\title{
Geometric Invariants of the Quantum Hall Effect
}

\section{Jingbo Xia *}

Department of Mathematics, State University of New York at Buffalo, Buffalo, New York 14214, USA

\begin{abstract}
We study the two-dimensional Hall effect with a random potential. The Hall conductivity is identified as a geometric invariant associated with an algebra of observables. Using the pairing between $K$-theory and cyclic cohomology theory, we identify this geometric invariant with a topological index, thereby giving the Hall conductivity a new interpretation.
\end{abstract}

\section{Introduction}

Since its experimental discovery [21], the quantum Hall effect [23] has attracted the attention of many researchers. The general approach in the theoretical work is that one considers a one-particle theory in a two-dimensional sample of infinite size. This leads to the study of the Hamiltonian

$$
H_{\omega}=\left[K_{x}^{2}+K_{y}^{2}\right] / 2+Q_{\omega}(x, y), \omega \in \Omega,
$$

with the commutation relation

$$
\left[K_{x}, K_{y}\right]=i \beta .
$$

Here, $\beta$ accounts for the presence of a magnetic field and $Q$ is a real function on the state space $\Omega$ which admits a flow of translation by $\mathbb{R}^{2}$. D. Thouless et al. [28] considered periodic potentials $Q$, i.e. the case where $\Omega$ is the two dimensional torus $T^{2}=\mathbb{R}^{2} / \mathbb{Z}^{2}$ with the natural flow induced by $\mathbb{R}^{2}$. They showed that with such a potential and a magnetic field of rational flux, the conductance of any filled, isolated band in the spectrum of the Hamiltonian is a topological invariant which is an integral multiple of $e^{2} / \hbar$. Since then substantial progress has been made on this subject through the contributions of many authors [1,2,3,5-8, 18,22]. Among these references, J. Bellissard's work has a close relation with this paper.

It was first observed in [5] that the two-dimensional Hall conductivity can be identified as the Chern character of the spectral projection of $H_{\omega}$ corresponding to $\left(-\infty, E_{F}\right)$, where $E_{F}$ is the Fermi level. This led to the introduction of A. Connes' geometry-operator algebra techniques to the study of the quantum Hall effect

* Supported in part by the National Science Foundation under Grant No. DMS-8717185 
$[6,7,8]$. In order to use these techniques, Bellissard constructed an algebra of observables $\mathscr{A}_{\omega}$ which contains sufficient information about the Hamiltonian $H_{\omega}$. It was noted in [5] that a computation of the current yields that the Hall conductivity $\sigma_{H}$ equals $\left(e^{2} / 2 \pi \hbar\right) \tau\left(P_{F}\left[\delta_{1}\left(P_{F}\right), \delta_{2}\left(P_{F}\right)\right]\right) / 2 \pi i$, where $\tau$ and $\delta_{1}, \delta_{2}$ are, respectively, a trace and two derivations on $\mathscr{A}_{\omega}$, and $P_{F}$ is the Fermi projection. (Also see $[3,22]$.) According to Connes [14], quantities of form $\tau\left(P\left[\delta_{1}(P), \delta_{2}(P)\right]\right)$ are in fact a geometric invariant. This invariant can also be interpreted as the pairing of $[P] \in K_{0}\left(\mathscr{A}_{\omega}\right)$ with a cyclic 2-cocycle $\psi$, in the sense of [15], on the algebra of observables. The cocycle gives rise to an element $[\psi]$ in the even cyclic cohomology group of a smooth subalgebra $\mathscr{A}_{\omega}^{\infty}$ which contains all the spectral projection of $H_{\omega}$ corresponding to gaps. Thus the proof of the quantization of the conductivity becomes the computation of all possible pairings $\langle[\psi],[P]\rangle$. We will follow Bellissard's formalism to the point of reducing the computation of the Hall conductivity to that of these pairings. But this is also the point at which [5-8] and our work take on divergent courses. Using a Fredholm module $(\mathscr{H}, G, F)$, Bellissard identified the Hall conductivity as an analytical index,

$$
\tau\left(P\left[\delta_{1}(P), \delta_{2}(P)\right]\right) / 2 \pi i=\operatorname{Ind} F_{P},
$$

where $F_{P}$ is a Fredholm operator. The importance of this formula is that it holds true for projections in the Sobolev space of $\mathscr{A}_{\omega}[6,7]$.

The purpose of this paper is to identify $\langle[\psi],[P]\rangle$ and, therefore, the Hall conductivity as a topological index which is obviously an integer. The technique required to achieve this is quite different from Bellissard's; we use the Thom isomorphisms for $C^{*}$-crossed products and the cyclic cohomology theory introduced by Connes. We write the Hamiltonian in the form of

$$
H_{\omega}=\left[-\partial^{2} / \partial x^{2}+(-i \partial / \partial y-\beta x)^{2}\right] / 2+Q_{\omega}(x, y) .
$$

This particular form of the Hamiltonian makes apparent that the corresponding algebra of observables $C_{\omega}^{*}\left(\Omega, \mathbb{R}^{2}, \beta\right)$ is an iterative crossed product of $C(\Omega)$ by two copies of $\mathbb{R}$. We use this fact to prove a labelling theorem for the gaps of the spectrum of $H_{\omega}$. Composing the two Thom isomorphisms involved here, one obtains a natural isomorphism $\phi: K_{0}(C(\Omega)) \rightarrow K_{0}\left(C_{\omega}^{*}\left(\Omega, \mathbb{R}^{2}, \beta\right)\right)$. Let $\mu$ be the ergodic measure that induces the trace $\tau$. It turns out that the pull back (in the sense of the pairing between cyclic cohomology theory and $K$-theory) of the cyclic cohomology class $[\psi]$ by the transpose of $\phi$ is the class $-[\mu]$. Since each element of $K_{0}(C(\Omega))$ is naturally identified with the difference of two classes of vector bundles, we can state our main result as follows.

Theorem. Assume that $\beta$ is a nonzero real number.

(i) We have $\langle[\psi],[P]\rangle=-\left\langle[\mu], \phi^{-1}[P]\right\rangle$ for smooth $[P] \in K_{0}\left(C^{*}\left(\Omega, \mathbb{R}^{2}, \beta\right)\right)$.

(ii) Given a real potential $Q \in C(\Omega)$, if the Fermi energy $E_{F}$ belongs to a gap of the spectrum of $H_{\omega}$, then there are vector bundles $V^{+}$and $V^{-}$on $\Omega$ such that $\phi^{-1}\left[P_{F}\right]=\left[V^{+}\right]-\left[V^{-}\right]$and such that the Hall conductivity equals

$$
\sigma_{H}=\left(e^{2} / 2 \pi \hbar\right)\left\langle[\psi],\left[P_{F}\right]\right\rangle=\left(\operatorname{dim} V^{-}-\operatorname{dim} V^{+}\right)\left(e^{2} / 2 \pi \hbar\right)
$$

The rest of the paper is organized as follows. In the first section, we discuss 
the algebra of observables associated with the random Hamiltonian and the cyclic cocycle which will later be used to express the conductivity. In Sect. 2, we prove the gap labelling theorem for the Hamiltonian $H_{\omega}$. This requires the identification of $C_{\omega}^{*}\left(\Omega, \mathbb{R}^{2}, \beta\right)$ with the iterative crossed product of $C(\Omega)$ by two copies of $\mathbb{R}$. As it turns out, this identification is crucial to the proof of our main result. We identify the conductivity with the geometric invariant $\left\langle[\psi],\left[P_{F}\right]\right\rangle$ in Sect. 3. Section 4 is proof of our main theorem.

\section{The Random Hamiltonian and the Algebra of Observables}

Throughout the paper, $\Omega$ will denote a separable, connected, compact Hausdorff space. It will be assumed that there is a group of homeomorphisms $\left\{\varphi_{x, y}:(x, y) \in \mathbb{R}^{2}\right\}$ acting on $\Omega$ such that for any $f \in C(\Omega),(x, y) \mapsto f \circ \varphi_{x, y}$ is a continuous map from $\mathbb{R}^{2}$ into $C(\Omega)$. For the sake of simplicity, $\varphi_{x, y}(\omega)$ will be denoted in the form of flow translation $\omega+(x, y)$. For any $f \in C(\Omega)$, we define $f_{\omega}(x, y)=f(\omega+(x, y)), \omega \in \Omega$, $(x, y) \in \mathbb{R}$. Let $d \mu$ denote a translation invariant, ergodic, probability measure of the flow $\left(\Omega, \varphi, \mathbb{R}^{2}\right)$. Given a real function $Q \in C(\Omega)$, one has a family of Hamiltonians

$$
H_{\omega}=\left[-\partial^{2} / \partial x^{2}+(-i \partial / \partial y-\beta x)^{2}\right] / 2+Q_{\omega}(x, y) \text { on } L^{2}\left(\mathbb{R}^{2}\right), \omega \in \Omega .
$$

Here, $\beta$ is a real number which arises from the physical system. In the case of the two-dimensional Hall effect with a uniform magnetic field $\mathbf{B}$ perpendicular to the $x y$-plane, the constant $\beta$ equals $e|\mathbf{B}| / \hbar c$. We will assume for the rest of the paper that $\beta$ is a nonzero real number. Under this assumption, the free Hamiltonian

$$
H_{0}=\left[-\partial^{2} / \partial x^{2}+(-i \partial / \partial y-\beta x)^{2}\right] / 2
$$

is in essence a linear oscillator. This can be seen in the following way. Let $K_{1}=-i \partial / \partial x$ and $K_{2}=-i \partial / \partial y-\beta x$. Let $\mathscr{F}: L^{2}\left(\mathbb{R}^{2}\right) \rightarrow L^{2}\left(\hat{\mathbb{R}}^{2}\right)$ be the Fourier transform $(\mathscr{F} f)(u, v)=\int_{\mathbb{R}^{2}} \exp [-2 \pi i(u x+v y)] f(x, y) d x d y$. Then on $L^{2}\left(\widehat{\mathbb{R}}^{2}\right)$, we have $\hat{K}_{1}=\mathscr{F} K_{1} \mathscr{F} *=2 \pi u, \quad \hat{K}_{2}=\mathscr{F} K_{2} \mathscr{F} *=2 \pi v-(\beta / 2 \pi) i \partial / \partial u$, and, therefore, $\hat{H}_{0}=$ $\mathscr{F} H_{0} \mathscr{F}^{*}=\left[(2 \pi u)^{2}+(2 \pi v-(\beta / 2 \pi) i \partial / \partial u)^{2}\right] / 2$. Let $U_{\beta}$ be the unitary operator of multiplication by $\exp \left(i(2 \pi)^{2} u v / \beta\right)$ on $L^{2}\left(\hat{\mathbb{R}}^{2}\right)$. Then

$$
\begin{aligned}
& \tilde{K}_{1}=U_{\beta} \hat{K}_{1} U_{\beta}^{*}=U_{\beta} \mathscr{F} K_{1} \mathscr{F} * U_{\beta}^{*}=2 \pi u=k_{1} \otimes 1, \\
& \tilde{K}_{2}=U_{\beta} \hat{K}_{2} U_{\beta}^{*}=U_{\beta} \mathscr{F} K_{2} \mathscr{F} * U_{\beta}^{*}=-(\beta / 2 \pi) i \partial / \partial u=k_{2} \otimes 1,
\end{aligned}
$$

where $k_{1}$ is the multiplication by $2 \pi u$ on $L^{2}(\mathbb{R})$ and $k_{2}=-(\beta / 2 \pi) i d / d u$ on the same space. Hence $H_{0}$ is unitarily equivalent to

$$
\begin{aligned}
\tilde{H}_{0} & =U_{\beta} \hat{H}_{0} U_{\beta}^{*}=U_{\beta} \mathscr{F} H_{0} \mathscr{F} * U_{\beta}^{*}=\left[-(\beta / 2 \pi)^{2} \partial^{2} / \partial u^{2}+(2 \pi u)^{2}\right] / 2 \\
& =2^{-1}\left[-(\beta / 2 \pi)^{2} d^{2} / d u^{2}+(2 \pi u)^{2}\right] \otimes 1 .
\end{aligned}
$$

In this paper, we use a function on a given $\Omega$ to describe the disorder associated with the physical system. We take this approach because of its mathematical convenience. On the other hand, if a potential $Q$ on $\mathbb{R}^{2}$ is given, one can think of $\Omega$ as the hull of $Q$ and the flow on $\Omega$ as that induced by translating $Q$ [5]. Oftentimes, one can assume that the flow $\left(\Omega, \varphi, \mathbb{R}^{2}\right)$ is minimal and uniquely ergodic. This is the case, for example, when the potential $Q$ is periodic, as was considered in [28]. 
For such a potential, $\Omega$ is the two-dimensional torus $T^{2} \cong \mathbb{R}^{2} / \mathbb{Z}^{2}$ and the flow is that induced by the group structure of $\Omega$.

Let $C_{c}\left(\Omega \times \mathbb{R}^{2}\right)$ denote the collection of continuous functions on $\Omega \times \mathbb{R}^{2}$ with compact support. For any $a \in C_{c}\left(\Omega \times \mathbb{R}^{2}\right)$ and $\omega \in \Omega$, let

$$
\left(\pi_{\omega}(a) f\right)(x, y)=\int_{\mathbb{R}^{2}} a(\omega+(x, y), \xi, \eta) \exp (-i \beta x \eta) f(x+\xi, y+\eta) d \xi d \eta, \quad f \in L^{2}\left(\mathbb{R}^{2}\right) .
$$

For a fixed $\omega$, the $C^{*}$-algebra generated by all $\pi_{\omega}(a), a \in C_{c}\left(\Omega \times \mathbb{R}^{2}\right)$, will be denoted by $C_{\omega}^{*}\left(\Omega, \mathbb{R}^{2}, \beta\right)$. This is the algebra of observables. If $\beta$ were 0 , this algebra would be a representation of the $C^{*}$-crossed product $C(\Omega) \times{ }_{\varphi} \mathbb{R}^{2}$. We will later identify $C_{\omega}^{*}\left(\Omega, \mathbb{R}^{2}, \beta\right)$ as an iterative crossed product of $C(\Omega)$ by two copies of $\mathbb{R}$. This identification is absolutely essential to our work. Although the algebra of observables constructed here has a different appearance from the algebra introduced by Bellissard in $[5,6,7]$, they are actually unitarily equivalent. We choose this particular representation of the algebra because of our need to identify it as a double crossed product.

For any $z \in \mathbb{C} \backslash \mathbb{R}$, the resolvent $\left(z-H_{\omega}\right)^{-1}$ belongs to $C_{\omega}^{*}\left(\Omega, \mathbb{R}^{2}, \beta\right)$. But this is not an obvious fact and a little explanation is in order. Using the usual power series expansion, one sees that it suffices to show that the resolvent of the free Hamiltonian, $\left(z-H_{0}\right)^{-1}$, belongs to $C_{\omega}^{*}\left(\Omega, \mathbb{R}^{2}, \beta\right)$. By $(1.3)$, the $C^{*}$-algebra $\mathscr{K}$ generated by all

$$
\int_{\mathbb{R}^{2}} b(\xi, \eta) \exp \left(i \xi K_{1}\right) \exp \left(i \eta K_{2}\right) d \xi d \eta, \quad b \in C_{c}\left(\mathbb{R}^{2}\right)
$$

is contained in $C_{\omega}^{*}\left(\Omega, \mathbb{R}^{2}, \beta\right)$. On the other hand, the $C^{*}$-algebra generated by

$$
\int_{\mathbb{R}^{2}} b(\xi, \eta) \exp \left(i \xi k_{1}\right) \exp \left(i \eta k_{2}\right) d \xi d \eta, \quad b \in C_{c}\left(\mathbb{R}^{2}\right)
$$

is $\mathscr{K}\left(L^{2}(\mathbb{R})\right)$, the collection of all the compact operators on $L^{2}(\mathbb{R})$. By $(1.1)$, the map that takes (1.4) to (1.5) extends to an isomorphism $\mathscr{J}_{\beta}$ from $\mathscr{K}$ onto $\mathscr{K}\left(L^{2}(\mathbb{R})\right)$. It is a well known fact that if $z$ is a nonreal number, $\left(z-\left(k_{1}^{2}+k_{2}^{2}\right) / 2\right)^{-1} \in \mathscr{K}\left(L^{2}(\mathbb{R})\right)$. Therefore $\left(z-\tilde{H}_{0}\right)^{-1} \in \mathscr{K}\left(L^{2}(\mathbb{R})\right) \otimes \mathbb{C}$. One deduces from this, (1.1) and (1.2) that $\left(z-H_{0}\right)^{-1} \in \mathscr{K} \subset C_{\omega}^{*}\left(\Omega, \mathbb{R}^{2}, \beta\right)$.

On the space $L^{2}\left(\Omega \times \mathbb{R}^{2}\right)$, where the measure is $d \mu \times d x d y$, let $C^{*}\left(\Omega, \mathbb{R}^{2}, \beta\right)$ be the $C^{*}$-algebra generated by twisted convolution operators

$$
\begin{gathered}
\left(C_{a} f\right)(\omega, x, y)=\int_{\mathbb{R}^{2}} a(\omega, \xi, \eta) \exp (-i \beta x \eta) f(\omega+(\xi, \eta), x+\xi, y+\eta) d \xi d \eta, \\
a \in C_{c}\left(\Omega \times \mathbb{R}^{2}\right), \quad f \in L^{2}\left(\Omega \times \mathbb{R}^{2}\right) .
\end{gathered}
$$

We call the function $a$ the kernal of $C_{a}$. It is easy to verify that the kernel of $\left(C_{a}\right)^{*}$ is

$$
a^{*}(\omega, x, y)=\exp (-i \beta x y) \overline{a(\omega+(x, y),-x,-y)} .
$$

For any $a, b \in C_{c}\left(\Omega \times \mathbb{R}^{2}\right), C_{a} C_{b}$ has a kernel

$$
a * b(\omega, x, y)=\int_{\mathbb{R}^{2}} a(\omega, \xi, \eta) \exp (-i \beta \xi(y-\eta)) b(\omega+(\xi, \eta), x-\xi, y-\eta) d \xi d \eta
$$


These formulas show that

$$
\tau\left(C_{a}\right)=\int_{\Omega} a(\omega, 0,0) d \mu(\omega), \quad a \in C_{c}\left(\Omega \times \mathbb{R}^{2}\right)
$$

defines a trace on $C^{*}\left(\Omega, \mathbb{R}^{2}, \beta\right)$. On $L^{2}\left(\Omega \times \mathbb{R}^{2}\right)$, define $\left(D_{1} f\right)(\omega, x, y)=2 \pi i x f(\omega, x, y)$ and $\left(D_{2} f\right)(\omega, x, y)=2 \pi i y f(\omega, x, y)$. These operators induce two derivations on $C^{*}\left(\Omega, \mathbb{R}^{2}, \beta\right)$ in the following way. For $A \in C^{*}\left(\Omega, \mathbb{R}^{2}, \beta\right)$, define

$$
\delta_{1}(A)=\left[A, D_{1}\right]=A D_{1}-D_{1} A \quad \text { and } \quad \delta_{2}(A)=\left[A, D_{2}\right]=A D_{2}-D_{2} A
$$

if these operators belong to $C^{*}\left(\Omega, \mathbb{R}^{2}, \beta\right)$. Obviously for every $a \in C_{c}\left(\Omega \times \mathbb{R}^{2}\right), C_{a}$ belongs to the domain of $\delta_{1}$ and $\delta_{2}$. The operators $\delta_{1}\left(C_{a}\right)$ and $\delta_{2}\left(C_{a}\right)$ have kernels $2 \pi i x a(\omega, x, y)$ and $2 \pi i y a(\omega, x, y)$ respectively. Let $C^{\infty}\left(\Omega, \mathbb{R}^{2}, \beta\right)$ be the collection of elements $A$ such that $\delta_{1}^{n}\left(\delta_{2}^{m}(A)\right) \in C^{*}\left(\Omega, \mathbb{R}^{2}, \beta\right)$, and such that $\tau\left(\delta_{1}^{n}\left(\delta_{2}^{m}(A)\right)^{*} \delta_{1}^{n}\left(\delta_{2}^{m}(A)\right)\right)<\infty$ for any nonnegative integers $n$ and $m$. The algebra $C^{\infty}\left(\Omega, \mathbb{R}^{2}, \beta\right)$ is a smooth subalgebra of $C^{*}\left(\Omega, \mathbb{R}^{2}, \beta\right)$ closed under analytic functional calculus [13]. According to [13, Page 52], we have

Proposition 1.1. The inclusion map $\imath: C^{\infty}\left(\Omega, \mathbb{R}^{2}, \beta\right) \rightarrow C^{*}\left(\Omega, \mathbb{R}^{2}, \beta\right)$ induces an isomorphism

$$
l_{*}: K_{0}\left(C^{\infty}\left(\Omega, \mathbb{R}^{2}, \beta\right)\right) \rightarrow K_{0}\left(C^{*}\left(\Omega, \mathbb{R}^{2}, \beta\right)\right)
$$

between the K-groups.

Let $\mathscr{W}=W^{*}\left(\Omega, \mathbb{R}^{2}, \beta\right)$ be the weak*-closure of $C^{*}\left(\Omega, \mathbb{R}^{2}, \beta\right)$ in $\mathscr{L}\left(L^{2}\left(\Omega \times \mathbb{R}^{2}\right)\right)$. It is known that the von Neumann algebra $\mathscr{W}$ is a factor if the action of $\mathbb{R}^{2}$ on $\Omega$ is free and it is of type- $\mathrm{II}_{\infty}$ if the orbits of the flow are not closed [14]. The trace $\tau$ naturally extends to a normal trace, which we denote by the same letter, on $\mathscr{W}$. One can easily deduce from formulas (1.6-1.9) that the GNS representation of $\mathscr{W}$ induced by $\tau$ is unitarily equivalent to the identity representation of $\mathscr{W}$ on $L^{2}\left(\Omega \times \mathbb{R}^{2}\right)$. Hence $\tau$ is always a faithful trace on $\mathscr{W}$. If $A \in \mathscr{W}$ is such that $\tau\left(A^{*} A\right)<\infty$, then $A$ has a square-integrable kernel $a$ on $\Omega \times \mathbb{R}^{2}$ in the sense that for any $f \in L^{2}\left(\Omega \times \mathbb{R}^{2}\right)$, Af is given by the right-hand side of (1.6) [20].

Let $A_{1}, A_{2} \in C^{*}\left(\Omega, \mathbb{R}^{2}, \beta\right)$ be two elements in the domain of $\delta_{1}$ and $\delta_{2}$ such that $\tau\left(A_{j}^{*} A_{j}\right)<\infty$ and $\tau\left(\delta_{k}\left(A_{j}\right) * \delta_{k}\left(A_{j}\right)\right)<\infty, j, k=1,2$. Then $\delta_{k}\left(A_{1} A_{2}\right)=\delta_{k}\left(A_{1}\right) A_{2}+$ $A_{1} \delta_{k}\left(A_{2}\right)$ is of $\tau$-trace class, $k=1,2$. Since $\delta_{k}\left(A_{j}\right)$ 's have square-integrable kernels, a simple computation using formulas (1.6), (1.8) and (1.9) yields $\tau\left(\delta_{k}\left(A_{1}\right) A_{2}\right)=$ - $\tau\left(A_{1} \delta_{k}\left(A_{2}\right)\right)$. Hence for $A_{1}$ and $A_{2}$ satisfying the above condition, we have

$$
\tau\left(\delta_{k}\left(A_{1} A_{2}\right)\right)=0, \quad k=1,2 .
$$

In other words these derivations annihilate the trace. Because $\delta_{1}$ and $\delta_{2}$ commute,

$$
\psi\left(a_{0}, a_{1}, a_{2}\right)=\tau\left(a_{0}\left[\delta_{1}\left(a_{1}\right) \delta_{2}\left(a_{2}\right)-\delta_{2}\left(a_{1}\right) \delta_{1}\left(a_{2}\right)\right]\right)
$$

defines a cyclic 2-cocycle on $C^{\infty}\left(\Omega, \mathbb{R}^{2}, \beta\right)[15]$.

Let $V$ be the unitary operator $(V f)(\omega, x, y)=f(\omega+(x, y), x, y)$ on $L^{2}\left(\Omega \times \mathbb{R}^{2}\right)$. It is straightforward to verify that for any $a \in C_{c}(\Omega \times \mathbb{R})$,

$$
V C_{a}=\int_{\Omega} \oplus \pi_{\omega}(a) d \mu(\omega) V,
$$


where the direct integral corresponds to the space decomposition

$$
L^{2}(\Omega \times \mathbb{R})=\int_{\Omega} \oplus L^{2}(\mathbb{R}) d \mu(\omega) .
$$

If the flow $\left(\Omega, \varphi, \mathbb{R}^{2}\right)$ is minimal, then $\left\|\pi_{\omega}(a)\right\|$ is independent of $\omega$ [20]. In this case, for any $\omega \in \Omega, C_{a} \mapsto \pi_{\omega}(a)$ extends to an isomorphism from $C^{*}\left(\Omega, \mathbb{R}^{2}, \beta\right)$ onto $C_{\omega}^{*}\left(\Omega, \mathbb{R}^{2}, \beta\right)$. If the flow is not minimal, one can still show, by using the ergodicity of $\mu$, that there is a Borel set $N \subset \Omega$ of measure 0 such that $\pi_{\omega}$ is an isomorphism for every $\omega \in \Omega \backslash N$. Let

$$
\mathbf{H}=V^{*} \int_{\Omega} \oplus H_{\omega} d \mu(\omega) V
$$

Then

$$
\begin{aligned}
(\mathbf{H} f)(\omega, x, y)= & {\left[-\partial^{2} / \partial s^{2}+(-i \partial / \partial t-\beta x)^{2}\right] f(\omega+(s, t), x+s, y+t) /\left.2\right|_{t=s=0} } \\
& +Q(\omega) f(\omega, x, y) .
\end{aligned}
$$

Thus we have

Proposition 1.2. (i) The map $\pi_{\omega}$ is an isomorphism from $C^{*}\left(\Omega, \mathbb{R}^{2}, \beta\right)$ onto $C_{\omega}^{*}\left(\Omega, \mathbb{R}^{2}, \beta\right)$ for a.e. $\omega \in \Omega$. If the flow is minimal, then every $\pi_{\omega}$ is an isomorphism.

(ii) Given a real potential $Q \in C(\Omega)$, for a.e. $\omega \in \Omega$, the spectrum of $H_{\omega}$ is the same as that of $\mathbf{H}$ defined by (1.12). If the flow is minimal, then every $H_{\omega}$ has the same spectrum as $\mathbf{H}$.

Because of the isomorphism $\pi_{\omega}$, for a.e. $\omega, \tau$ will be regarded as a trace on $C_{\omega}^{*}\left(\Omega, \mathbb{R}^{2}, \beta\right)$. Similarly, $\delta_{1}$ and $\delta_{2}$ are regarded as derivations on $C_{\omega}^{*}\left(\Omega, \mathbb{R}^{2}, \beta\right)$ and $\psi$ defined by $(1.11)$ as a cyclic cocycle on $C_{\omega}^{\infty}\left(\Omega, \mathbb{R}^{2}, \beta\right)=\pi_{\omega}\left(C^{\infty}\left(\Omega, \mathbb{R}^{2}, \beta\right)\right)$.

Lemma 1.3. Let $G$ be the resolvent set of $H_{\omega}$ and let $R_{\omega}(z)=\left(z-H_{\omega}\right)^{-1}$. Then $K_{j} R_{\omega}(z), j=1,2$, are $C_{\omega}^{*}\left(\Omega, \mathbb{R}^{2}, \beta\right)$-valued analytic functions on $G$ and for any $z \in G$, $\tau\left(R_{\omega}(z)^{*} R_{\omega}(z)\right)<\infty$.

Proof. Let $R_{0}(z)=\left(z-H_{0}\right)^{-1}$, then for nonreal $z, R_{\omega}(z)=R_{0}(z)+R_{0}(z) Q_{\omega} R_{\omega}(z)$ and, therefore, $K_{j} R_{\omega}(z)=K_{j} R_{0}(\lambda)+K_{j} R_{0}(z) Q_{\omega} R_{\omega}(z)$. Let $S=\{(n+1 / 2)|\beta|: n=$ $0,1,2, \ldots\}$, the spectrum of $H_{0}$ [25]. We first show that, off the set $S, K_{j} R_{0}(z)$ belongs to $C_{\omega}^{*}\left(\Omega, \mathbb{R}^{2}, \beta\right)$. According to (1.1) and (1.2), this will follow if we can show that for $z \in \mathbb{C} \backslash S, k_{j}\left(z-\left(k_{1}^{2}+k_{2}^{2}\right) / 2\right)^{-1}$ is a compact operator on $L^{2}(\mathbb{R})$. Let $p=k_{1}+i k_{2}$. Then, by the commutation relation $\left[k_{1}, k_{2}\right]=i \beta$, we have $p^{*} p=k_{1}^{2}+$ $k_{2}^{2}-\beta$. Since $\left(\bar{z}-\left(k_{1}^{2}+k_{2}^{2}\right) / 2\right)^{-1} p^{*} p\left(z-\left(k_{1}^{2}+k_{2}^{2}\right) / 2\right)^{-1}$ is a compact operator, by polar decomposition, $p\left(z-\left(k_{1}^{2}+k_{2}^{2}\right) / 2\right)^{-1}$ is compact. A similar argument shows that $p^{*}\left(z-\left(k_{1}^{2}+k_{2}^{2}\right) / 2\right)^{-1}$ is also compact. Hence $k_{j}\left(z-\left(k_{1}^{2}+k_{2}^{2}\right) / 2\right)^{-1}, j=1,2$, are compact operators. Once this has been established, the analyticity of the map $z \mapsto K_{j} R_{0}(z)$ is obvious. Hence $K_{j} R_{\omega}(z)$ is a $C_{\omega}^{*}\left(\Omega, \mathbb{R}^{2}, \beta\right)$-valued analytic function on $G \backslash S$. Since $S$ has no points of accumulation and since $\left\langle K_{j} R_{\omega}(z) f, g\right\rangle=$ $\left\langle R_{\omega}(z) f, K_{j} g\right\rangle$ for any $f \in L^{2}\left(\mathbb{R}^{2}\right)$ and $g$ in the domain of $K_{j}$, all the singularities of $K_{j} R_{\omega}(z)$ in $G$ are removable.

By the resolvent identity we used in the previous paragraph and the Cauchy integral formula, in order to show $\tau\left(R_{\omega}(z)^{*} R_{\omega}(z)\right)<\infty$ on $G$, it suffices to show $\tau\left(R_{0}(z)^{*} R_{0}(z)\right)<\infty$ on $\mathbb{C} \backslash S$. Since the multiplicity of $\left(k_{1}^{2}+k_{2}^{2}\right) / 2$ at every point of 
$S$ is $1[16],\left(z-\left(k_{1}^{2}+k_{2}^{2}\right) / 2\right)^{-1}=\mathscr{J}_{\beta}\left(R_{0}(z)\right)$ is a Hilbert-Schmidt operator on $L^{2}(\mathbb{R})$ whenever $z \in \mathbb{C} \backslash S$. By (1.3),(1.4),(1.8) and a simple computation, one finds that an element $A \in \mathscr{K}$ is of $\tau$-trace class if and only if $\mathscr{J}_{\beta}(A)$ is a trace class operator on $L^{2}(\mathbb{R})$ and that

$$
\tau(A)=(|\beta| / 2 \pi) \operatorname{tr}\left(\mathscr{J}_{\beta}(A)\right)
$$

Hence $\tau\left(R_{0}(z)^{*} R_{0}(z)\right)<\infty$.

Remark. The key in the above proof is that the singularities of the analytic function $K_{j} R_{0}(z)$ are isolated points in $\mathbb{C}$ if $\beta \neq 0$. If $\beta=0$, however, $K_{j} R_{0}(z)$ does not have analytic continuation on $[0, \infty)$ and the proof of the above lemma will fail. Consequently, the following proposition may not be true. This is the why we must assume $\beta \neq 0$.

Proposition 1.4. Let $\lambda$ be a real number not in the spectrum of $H_{\omega}$ and let $P$ be the spectral projection of $H_{\omega}$ corresponding to the interval $(-\infty, \lambda)$. Then

$$
P \in C_{\omega}^{\infty}\left(\Omega, \mathbb{R}^{2}, \beta\right) .
$$

Proof. Since the spectrum of $H_{\omega}$ in $(-\infty, \lambda)$ is contained in some finite interval $(b, \lambda)$, we can choose a counter-clockwise oriented contour $\Gamma \subset G$ which encircles $(b, \lambda)$ once. Thus $P=\int_{\Gamma} R_{\omega}(z) d z / 2 \pi i$. Because $\delta_{j}\left(R_{\omega}(z)\right)=2 \pi R_{\omega}(z) K_{j} R_{\omega}(z)$, it follows from Lemma 1.3 that $\delta_{j}(P)=-i \int_{\Gamma} R_{\omega}(z) K_{j} R_{\omega}(z) d z \in C_{\omega}^{*}\left(\Omega, \mathbb{R}^{2}, \beta\right)$. Formally, we have $\delta_{j}\left(K_{j}\right)=2 \pi$ and $\delta_{j}\left(K_{k}\right)=0$ for $j \neq k$. Hence that $\delta_{j}^{n}\left(\delta_{k}^{m}(P)\right)$ belongs to $C_{\omega}^{*}\left(\Omega, \mathbb{R}^{2}, \beta\right)$ can be proved by mathematical induction and repeated use of Lemma 1.3.

\section{The Integrated Density of States and the Fermi Energy}

Let $\mathbf{H}=\int_{\mathbb{R}} \lambda d \mathbf{P}_{\lambda}$ be the spectral decomposition of the operator (1.12). Since the resolvent $(z-\mathbf{H})^{-1}$ belongs to the ideal generated by the $\tau$-trace class operators in $\mathscr{W}$, every spectral projection $\mathbf{P}(-\infty, \lambda), \lambda<\infty$, is finite in $\mathscr{W}$. As usual, the increasing function $N_{Q}(\lambda)=\tau(\mathbf{P}(-\infty, \lambda))$ is called the integrated density of states (IDS) for the potential $Q$. Since $\tau$ is a faithful trace, the spectrum of $\mathbf{H}$ and, for a.e. $\omega$, that of $H_{\omega}$, is the complement in $\mathbb{R}$ of the open intervals on which $N_{Q}$ is a constant. If the action of $\mathbb{R}^{2}$ action on $\Omega$ is uniquely ergodic, then $N_{Q}(\lambda)$ can be recovered from the limit distribution of the eigenvalues of $H_{\omega}$ restricted to finite domains of $\mathbb{R}^{2}$ (hence the term IDS). In the case $\beta=0$, this was done by M. Shubin in [27]. One can trace the steps of [27] to reproduce such a result for the case $\beta \neq 0$. But since this is not essential to our investigation, we omit the details.

Like the Hamiltonians for many other disordered systems, the function $N_{Q}$ provides a labelling for the gaps of the spectrum of $H_{\omega}$ [4]. A commonly used method for determining all the possible labels is to compute the image under the trace map of the $K_{0}$-group of the algebra of observables. For this purpose we will express $C^{*}\left(\Omega, \mathbb{R}^{2}, \beta\right)$ as an iterative $C^{*}$-crossed product of $C(\Omega)$ by two copies of $\mathbb{R}$. This idea comes from a careful inspection of (1.8). For the validity of the following 
constructions of $C^{*}$-crossed product algebras, the reader is referred to [26, Sect. 7.7].

From the group $\left\{\varphi_{x, y}:(x, y) \in \mathbb{R}^{2}\right\}$, one obtains two one-parameter groups of homeomorphisms on $\Omega$ :

$$
\theta_{t}(\omega)=\varphi_{0, t}(\omega), \quad \text { and } \quad \alpha_{t}(\omega)=\varphi_{t, 0}(\omega), \quad \omega \in \Omega, \quad t \in \mathbb{R} .
$$

The $C^{*}$-crossed product $C(\Omega) \times{ }_{\theta} \mathbb{R}$ of $C(\Omega)$ by the one-parameter group $\theta$ can be represented on $L^{2}(\Omega \times \mathbb{R})$ as follows: For $a \in C_{c}(\Omega \times \mathbb{R})$, and $f \in L^{2}(\Omega \times \mathbb{R})$, let

$$
(a * f)(\omega, y)=\int_{\mathbb{R}} a(\omega, t) f(\omega+(0, t), y+t) d t .
$$

Then for any $a, b \in C_{c}(\Omega \times \mathbb{R})$, we have

$$
(a * b)(\omega, y)=\int_{\mathbb{R}} a(\omega, t) b(\omega+(0, t), y-t) d t=\int_{\mathbb{R}} a(\omega, t) b\left(\theta_{t}(\omega), y-t\right) d t .
$$

The algebra $C(\Omega) \times{ }_{\theta} \mathbb{R}$ is the $C^{*}$-algebra generated by all $a \in C_{c}(\Omega \times \mathbb{R})$ with the algebraic structure described above. The invariant measure $d \mu$ gives rise to a trace $\tau_{1}$ on $C(\Omega) \times{ }_{\theta} \mathbb{R}:$

$$
\tau_{1}(a)=\int_{\Omega} a(\omega, 0) d \mu(\omega) .
$$

Let us now define a one-parameter group of automorphisms $\left\{\gamma_{s}: s \in \mathbb{R}\right\}$ on $C(\Omega) \times{ }_{\theta} \mathbb{R}$. For an $a \in C_{c}(\Omega \times \mathbb{R})$, let

$$
\gamma_{s}(a)(\omega, y)=\exp (-i \beta s y) a\left(\alpha_{s}(\omega), y\right)=\exp (-i \beta s y) a(\omega+(s, 0), y) .
$$

It is straightforward to verify that each $\gamma_{s}$ is an automorphism. Indeed $\gamma_{s}(a)=$ $U_{s}^{*} a U_{s}$, where $\left(U_{s} f\right)(\omega, y)=\exp (-i \beta s y) f(\omega+(s, 0), y)$ on $L^{2}(\Omega \times \mathbb{R})$. The $C^{*}$ crossed product $\left[C(\Omega) \times{ }_{\theta} \mathbb{R}\right] \times{ }_{\gamma} \mathbb{R}$ on $L^{2}((\Omega \times \mathbb{R}) \times \mathbb{R})$ is the norm-closure of $C_{c}\left(\mathbb{R}, C(\Omega) \times{ }_{\theta} \mathbb{R}\right)$, the collection of compactly supported, $C(\Omega) \times{ }_{\theta} \mathbb{R}$-valued continuous functions on $\mathbb{R}$. If we view $C_{c}(\Omega \times \mathbb{R})$ as a dense subalgebra of $C(\Omega) \times{ }_{\theta} \mathbb{R}$ in the sense of $(2.1)$, then $\left[C(\Omega) \times{ }_{\theta} \mathbb{R}\right] \times{ }_{\gamma} \mathbb{R}$ is generated by

$$
\left(\tilde{C}_{a} f\right)(\omega, y, x)=\int_{\mathbb{R}} \exp (-i \beta s y)\left(\int_{\mathbb{R}} a(s, \omega, t) f(\omega+(s, t), y+t, x+s) d t\right) d s,
$$

where $a \in C_{c}\left(\mathbb{R}, C_{c}(\Omega \times \mathbb{R})\right)=C_{c}(\mathbb{R} \times \Omega \times \mathbb{R})$ and $f \in L^{2}\left(\left(\Omega \times \mathbb{R}_{y}\right) \times \mathbb{R}_{x}\right)$. Furthermore, for any $a, b \in C_{c}\left(\mathbb{R}, C_{c}(\Omega \times \mathbb{R})\right), \widetilde{C}_{a} \widetilde{C}_{b}$ has a kernel

$$
(a * b)(x, \omega, t)=\int_{\mathbb{B}} \exp (-i \beta s(y-t))\left(\int_{\mathbb{B}} a(s, \omega, t) b(x-s, \omega+(s, t), y-t) d t\right) d s .
$$

Naturally, the trace on $\left[C(\Omega) \times{ }_{\theta} \mathbb{R}\right] \times{ }_{\gamma} \mathbb{R}$ is

$$
\tau\left(\widetilde{C}_{a}\right)=\int_{\Omega} a(0, \omega, 0) d \mu(\omega) .
$$

For $a \in C_{c}\left(\Omega \times \mathbb{R}^{2}\right)$, let $\tilde{a}(x, \omega, y)=a(\omega, x, y)$. A comparison of (1.6) and (1.8) with (2.5) and (2.6) shows that $C_{a} \mapsto \widetilde{C}_{\tilde{a}}$ extends to an isomorphism from $C^{*}\left(\Omega, \mathbb{R}^{2}, \beta\right)$ onto $\left[C(\Omega) \times{ }_{\theta} \mathbb{R}\right] \times{ }_{\gamma} \mathbb{R}$. Hence we have proved

Proposition 2.1. There is a natural isomorphism $\mathscr{I}: C^{*}\left(\Omega, \mathbb{R}^{2}, \beta\right) \rightarrow\left[C(\Omega) \times{ }_{\theta} \mathbb{R}\right] \times{ }_{\gamma} \mathbb{R}$ which respects the traces. 
For the rest of the paper, we will identify these algebras. Thus $\delta_{1}$ and $\delta_{2}$ will be regarded as derivations on $\left[C(\Omega) \times{ }_{\theta} \mathbb{R}\right] \times{ }_{\gamma} \mathbb{R}$ and $\psi$ as a cyclic cocycle on this algebra.

\section{Theorem 2.2.}

$$
\tau_{*}\left(K_{0}\left(C^{*}\left(\Omega, \mathbb{R}^{2}, \beta\right)\right)\right)=\tau_{*}\left(K_{0}\left(C(\Omega) \times{ }_{\varphi} \mathbb{R}^{2}\right)\right)+(\beta / 2 \pi) \mathbb{Z} .
$$

Proof. By (1.13), we have $(\beta / 2 \pi) \mathbb{Z}=\tau_{*}\left(K_{0}(\mathscr{K})\right) \subset \tau_{*}\left(K_{0}\left(C^{*}\left(\Omega, \mathbb{R}^{2}, \beta\right)\right)\right)$. For the rest of the proof, we need Connes' formula for the image of $K_{0}\left(\left[C(\Omega) \times{ }_{\theta} \mathbb{R}\right] \times{ }_{\gamma} \mathbb{R}\right)$ under the trace map. According to [13, Corollary 1], $\tau_{*}\left(K_{0}\left(\left[C(\Omega) \times{ }_{\theta} \mathbb{R}\right] \times{ }_{\gamma} \mathbb{R}\right)\right)$ consists of numbers

$$
\left(\tau_{1} \otimes \operatorname{tr}_{n}\right)\left(\delta_{\gamma}(u) u^{-1}\right) / 2 \pi i
$$

where $\delta_{\gamma}(a)=\lim _{s \rightarrow 0}\left(\gamma_{s}(a)-a\right) / s$ on $C(\Omega) \times{ }_{\theta} \mathbb{R}$ and $u$ is any smooth invertible element in $M_{n} \otimes C(\Omega) \times{ }_{\theta} \mathbb{R}^{\sim}$. By the definition (2.4) of $\gamma_{s}$, we have $\delta_{\gamma}=\delta_{\alpha}+\delta_{\beta}$, where, for an $a \in C_{c}(\Omega \times \mathbb{R})$ smooth along the orbits of $\mathbb{R}, \delta_{\alpha}(a)(\omega, y)=d a\left(\alpha_{s}(\omega), y\right) /\left.d s\right|_{s=0}$ and $\delta_{\beta}(a)(\omega, y)=-i \beta y a(\omega, y)$. Because $\quad\left[C(\Omega) \times{ }_{\theta} \mathbb{R}\right] \times{ }_{\alpha} \mathbb{R}=C(\Omega) \times{ }_{\varphi} \mathbb{R}$, by $[13]$, $\left(\tau_{1} \otimes \operatorname{tr}_{n}\right)\left(\delta_{\alpha}(u) u^{-1}\right) / 2 \pi i \in \tau_{*}\left(K_{0}\left(\left[C(\Omega) \times{ }_{\theta} \mathbb{R}\right] \times{ }_{\alpha} \mathbb{R}\right)\right)=\tau_{*}\left(K_{0}\left(C(\Omega) \times{ }_{\varphi} \mathbb{R}^{2}\right)\right)$. On the other hand, it is obvious that $\delta_{\beta}=(-\beta / 2 \pi) \delta_{l}$, where $\delta_{l}$ is the derivation induced by the one-parameter group of automorphisms $l_{s}(a)(\omega, y)=\exp (2 \pi i s y) a(\omega, y)$. Since $\left(\tau_{1} \otimes \operatorname{tr}_{n}\right)\left(\delta_{t}(u) u^{-1}\right) / 2 \pi i \in \tau_{*}\left(K_{0}\left(\left[C(\Omega) \times{ }_{\theta} \mathbb{R}\right] \times{ }_{t} \mathbb{R}\right)\right)$, which is known to be $\mathbb{Z}[13]$, we have $\left(\tau_{1} \otimes \operatorname{tr}_{n}\right)\left(\delta_{\beta}(u) u^{-1}\right) / 2 \pi i \in(\beta / 2 \pi) \mathbb{Z}$. Hence $\tau_{*}\left(K_{0}\left(\left[C(\Omega) \times{ }_{\theta} \mathbb{R}\right] \times{ }_{\gamma} \mathbb{R}\right)\right) \subset$ $\tau_{*}\left(K_{0}\left(C(\Omega) \times{ }_{\varphi} \mathbb{R}^{2}\right)\right)+(\beta / 2 \pi) \mathbb{Z}$. The inclusion the other way follows from the fact that for a smooth, invertible $u,\left(\tau_{1} \otimes \operatorname{tr}_{n}\right)\left(\delta_{\alpha}(u) u^{-1}\right) / 2 \pi i \in\left(\tau_{1} \otimes \operatorname{tr}_{n}\right)\left(\delta_{\gamma}(u) u^{-1}\right) / 2 \pi i+$ $(\beta / 2 \pi) \mathbb{Z} \subset \tau_{*}\left(K_{0}\left(\left[C(\Omega) \times{ }_{\theta} \mathbb{R}\right] \times{ }_{\gamma} \mathbb{R}\right)\right)$.

Corollary 2.3. If $\lambda$ belongs to a gap of the spectrum of $H_{\omega}$ for a.e. $\omega$, then

$$
N_{Q}(\lambda) \in \tau_{*}\left(K_{0}\left(C(\Omega) \times{ }_{\varphi} \mathbb{R}^{2}\right)\right)+(\beta / 2 \pi) \mathbb{Z}
$$

The computation of $\tau_{*}\left(K_{0}\left(C(\Omega) \times{ }_{\varphi} \mathbb{R}^{2}\right)\right)$ is contained in Connes' work [14]. He showed, for example, that if $\Omega$ is a smooth manifold and if the action $\varphi$ is generated by two smooth vector fields $X$ and $Y$, then $\tau_{*}\left(K_{0}\left(C(\Omega) \times{ }_{\varphi} \mathbb{R}^{2}\right)\right)$ consists of numbers $\langle C, \operatorname{ch}[e]\rangle$, where $\operatorname{ch}: K_{0}(C(\Omega)) \rightarrow H^{2}(\Omega, \mathbb{R})$ is the Chern character and $C$ is the Ruelle-Sullivan current of the foliation of $\Omega$ by the orbits of $\mathbb{R}^{2}$. Hence there is a large class of $\Omega$ and $\beta$ for which $\tau_{*}\left(K_{0}\left(C^{*}\left(\Omega, \mathbb{R}^{2}, \beta\right)\right)\right)$ is a dense subset of $\mathbb{R}$. Because of this density, it is not unreasonable to speculate that the gaps of $H_{\omega}$ may not always correspond to Landau levels and that the Hall conductivity may not always be identified with Landau band index. We will discuss this point further in the next section.

Recall that the Fermi energy of the physical system can be computed from $N_{Q}$ [27]. Let $M_{Q}$ be the "inverse" of $N_{Q}$, i.e.

$$
M_{Q}(t)=\sup \left\{\lambda: N_{Q}(\lambda)<t\right\} .
$$

If $\rho>0$ is the electron density, then we can express the Fermi energy as the average value of $M_{Q}$ on the interval $[0, \rho]$, that is

$$
E_{F}=E_{F}(\rho)=\rho^{-1} \int_{0}^{\rho} M_{Q}(t) d t
$$


This formula tells us that if the Fermi energy belongs to a gap of the spectrum, it will remain so if the electron density varies a small amount. If the spectrum of $H_{\omega}$ is a Cantor set, a small variation of the electron density could move the Fermi energy into a gap.

\section{The Conductivity and Cyclic Cohomology}

Assume that the Fermi energy $E_{F}$ for $H_{\omega}$ lies in a gap of its spectrum. We will denote by $P_{F}$ the spectral projection of $H_{\omega}$ corresponding to the interval $\left(-\infty, E_{F}\right)$. As was explained in $[5,6]$, the Hall conductivity $\sigma_{H}$ can be measured in the following way. Assuming that there is an electric field $\mathbf{E}$ in the direction of the Hall potential difference at time $t=0$, one calculates the evolution of the current as the electric field is being turned off. This involves taking the limits $|\mathbf{E}| \rightarrow 0$ and $t \rightarrow \infty$. A recent result of J. Avron, R. Seiler and L. Yaffe [3] allows the interchange of the order of these limits. Therefore the Kubo-Greenwood formula yields that at temperature 0 , the Hall conductivity equals

$$
\sigma_{H}=\lim _{\varepsilon \downarrow 0} \frac{e^{2}}{i \hbar \varepsilon} \int_{0}^{\infty} e^{-\varepsilon t} \tau\left(P_{F}\left[K_{1}, \exp \left(-i t H_{\omega}\right) K_{2} \exp \left(i t H_{\omega}\right)\right] P_{F}\right) d t .
$$

The same formula was used by H. Kunz [22].

Note that $\exp \left(i t H_{\omega}\right)$ is a multiplier of $C_{\omega}^{*}\left(\Omega, \mathbb{R}^{2}, \beta\right)$. Indeed it is fairly obvious that for any $z \in \mathbb{C} \backslash \mathbb{R}$ and $A \in C_{\omega}^{*}\left(\Omega, \mathbb{R}^{2}, \beta\right)$, exp $\left(i t H_{\omega}\right) R_{\omega}(z) A \in C_{\omega}^{*}\left(\Omega, \mathbb{R}^{2}, \beta\right)$. Hence that $\exp \left(i t H_{\omega}\right) A$ belongs to $C_{\omega}^{*}\left(\Omega, \mathbb{R}^{2}, \beta\right)$ follows from the resolvent identity $R_{\omega}(z)=R_{0}(z)+R_{0}(z) Q_{\omega} R_{\omega}(z)$ and the fact $\lim \left\|\lambda i R_{0}(i \lambda) A-A\right\|=0$, which can be easily derived from the structure of $C_{\omega}^{*}\left(\Omega, \mathbb{R}^{2}, \beta\right)$. Lemma 1.3 say that $K_{j} P_{F}$ and $P_{F} K_{j}, j=i, 2$, are of $\tau$-trace class in $C_{\omega}^{*}\left(\Omega, \mathbb{R}^{2}, \beta\right)$. Thus the integrand on the right-hand side of (3.1) is well defined. We will now rewrite $\sigma_{H}$ in terms of the cyclic cocycle (1.11).

Lemma 3.1. Let $P$ be the spectral projection of $H_{\omega}$ corresponding to $(-\infty, \lambda)$, where $\lambda$ belongs to a gap of the spectrum of $H_{\omega}$. Then

$$
\operatorname{Lim}_{\varepsilon \downarrow 0} \int_{0}^{\infty} e^{-\varepsilon t} \tau\left(P\left[K_{1}, \exp \left(-i t H_{\omega}\right) K_{2} \exp \left(i t H_{\omega}\right)\right] P\right) d t / \varepsilon=\tau\left(P\left[\delta_{1}(P), \delta_{2}(P)\right]\right) / 4 \pi^{2}
$$

Proof. Let

$$
A_{\varepsilon}=\int_{0}^{\infty} e^{-\varepsilon t} \exp \left(-i t H_{\omega}\right) K_{2} \exp \left(i t H_{\omega}\right) d t
$$

Since the trace $\tau$ has the property $\tau\left(B^{*}\right)=\overline{\tau(B)}$, we have $\operatorname{Im} \tau\left(P K_{1} P A_{\varepsilon} P\right)=$ $-i\left[\tau\left(P K_{1} P A_{\varepsilon} P\right)-\tau\left(P A_{\varepsilon} P K_{1} P\right)\right] / 2=0$. Therefore, the left-hand side of (3.2) equals

$$
\lim _{\varepsilon \downarrow 0} 2 i \operatorname{Im} \tau\left(P K_{1} A_{\varepsilon} P\right) / \varepsilon=\lim _{\varepsilon \downarrow 0} 2 i \operatorname{Im} \tau\left(P K_{1}\left[A_{\varepsilon}, P\right] P\right) / \varepsilon .
$$

Let $H_{\omega}=\int_{\mathbb{B}} \xi d P_{\xi}$ be the spectral decomposition. Then $P=P_{\lambda}$ and 


$$
\begin{aligned}
& {\left[A_{\varepsilon}, P\right]=\iint_{\mathbb{R}}\left(\int_{0}^{\infty} e^{-\varepsilon t} e^{i t(\eta-\xi)}\left[\chi_{(-\infty, \lambda)}(\eta)-\chi_{(-\infty, \lambda)}(\xi)\right] d t\right) d P_{\xi} K_{2} d P_{\eta}} \\
& =\iint_{\mathbb{R} \mathbb{R}} \frac{\chi_{(-\infty, \lambda)}(\eta)-\chi_{(-\infty, \lambda)}(\xi)}{\varepsilon+i(\xi-\eta)} d P_{\xi} K_{2} d P_{\eta} .
\end{aligned}
$$

Suppose that the spectrum of $H_{\omega}$ is contained in $(a+1, \infty)$. Let $\Gamma$ be a counter-clockwise oriented contour in $\mathbb{C}$ that encircles the interval $(a+1, \lambda)$ once and that crosses the real axis at $a$ and $\lambda$. If $\xi, \eta \in(a,+\infty) \backslash\{\lambda\}$, we have

$$
\frac{\chi_{(-\infty, \lambda)}(\eta)-\chi_{(-\infty, \lambda)}(\xi)}{\varepsilon+i(\xi-\eta)}=\frac{1}{2 \pi} \int_{\Gamma} \frac{d z}{(z-\xi)(z-\eta-i \varepsilon)} .
$$

Using the identity $R_{\omega}(z-i \varepsilon)=R_{\omega}(z)+i \varepsilon R_{\omega}(z) R_{\omega}(z-i \varepsilon)$ and the identity above, we have

$$
\begin{aligned}
{\left[A_{\varepsilon}, P\right] } & =\int_{\Gamma} R_{\omega}(z) K_{2} R_{\omega}(z-i \varepsilon) d z / 2 \pi \\
& =i \delta_{2}(P) / 2 \pi-\varepsilon \int_{\Gamma} R_{\omega}(z) K_{2} R_{\omega}(z) R_{\omega}(z-i \varepsilon) d z / 2 \pi i .
\end{aligned}
$$

Since $K_{j} \delta_{k}(P) \in C^{*}\left(\Omega, \mathbb{R}^{2}, \beta\right), \delta_{j}(P)$ is self-adjoint, and $P=P^{2}$, by (1.10), we have $\operatorname{Im} \tau\left(-i P K_{1} \delta_{2}(P) / 2 \pi\right)=\operatorname{Re} \tau\left(P K_{1} \delta_{2}(P) / 2 \pi\right)=\tau\left(P K_{1} \delta_{2}(P)+\delta_{2}(P) K_{1} P\right) / 2 \pi=$ $\tau\left(\delta_{2}\left(P K_{1} P\right)\right) / 2 \pi=0$. Hence

$$
\begin{aligned}
\operatorname{LHS} \text { of }(3.2) & =-\lim _{\varepsilon \downarrow 0} 2 i \operatorname{Im}\left[\int_{\Gamma} \tau\left(P K_{1} R_{\omega}(z) K_{2} R_{\omega}(z) R_{\omega}(z-i \varepsilon) P\right) d z / 2 \pi i\right] \\
& =-2 i \operatorname{Im}\left[\int_{\Gamma} \tau\left(P K_{1} R_{\omega}(z) K_{2} R^{2}(z) P\right) d z / 2 \pi i\right] \\
& =-2 i \operatorname{Im}\left[\int_{\Gamma} \tau\left(P R_{\omega}(z) K_{1} R_{\omega}(z) K_{2} R_{\omega}(z) P\right) d z / 2 \pi i\right] .
\end{aligned}
$$

Let $\zeta$ be a contour which is slightly larger than $\Gamma$ and which still crosses the real axis through the gap containing $\lambda$. Then

$$
\delta_{1}(P) \delta_{2}(P)=\left(-i \int_{\Gamma} R_{\omega}(z) K_{1} R_{\omega}(z) d z\right)\left(-i \int_{\zeta} R_{\omega}(u) K_{2} R_{\omega}(u) d u\right) .
$$

For $z \in \Gamma$ and $u \in \zeta$, we have $R_{\omega}(z) R_{\omega}(u)=\left(R_{\omega}(z)-R_{\omega}(u)\right) /(u-z)$. Hence

$$
P \delta_{1}(P) \delta_{2}(P) P=-\int_{\Gamma \zeta} P R_{\omega}(z) K_{1} \frac{R_{\omega}(z)-R_{\omega}(u)}{u-z} K_{2} R_{\omega}(u) P d z d u .
$$

Using the spectral decomposition of $H_{\omega}$ and the standard contour integral tricks, one finds $\int_{\Gamma} R_{\omega}(z)(u-z)^{-1} d z=2 \pi i P R_{\omega}(u)$ and $\int_{\zeta} R_{\omega}(u)(u-z)^{-1} d z=2 \pi i R_{\omega}(z)(1-P)$. Thus (3.5) yields

$$
P \delta_{1}(P) \delta_{2}(P) P=2 \pi i \int_{\zeta} P R_{\omega}(u) K_{1} R_{\omega}(u) K_{2} R_{\omega}(u) P d u .
$$

Comparing this with (3.4), we obtain 
LHS of $(3.2)=2 i \operatorname{Im} \tau\left(P \delta_{1}(P) \delta_{2}(P) /(2 \pi)^{2}\right)$

$$
=\tau\left(P \delta_{1}(P) \delta_{2}(P)-\delta_{2}(P) \delta_{1}(P) P\right) / 4 \pi^{2}=\tau\left(P\left[\delta_{1}(P), \delta_{2}(P)\right]\right) / 4 \pi^{2},
$$

as was to be proved.

Corollary 3.2. The Hall conductivity can be expressed as

$$
\sigma_{H}=\left(e^{2} / 2 \pi \hbar\right) \tau\left(P_{F}\left[\delta_{1}\left(P_{F}\right), \delta_{2}\left(P_{F}\right)\right]\right) / 2 \pi i .
$$

Quantities of form $\tau\left(P\left[\delta_{1}(P), \delta_{2}(P)\right]\right) / 2 \pi i$ were first studeid and recognized as a geometric invariant in the irrational rotation algebra by A. Connes [14]. Here, as it was the case in [14], we can identify this invariant as the "integral" of the curvature of a connection associated with the algebra of observables. Given a projection $P \in C_{\omega}^{\infty}\left(\Omega, \mathbb{R}^{2}, \beta\right), \mathscr{E}_{P}=P C_{\omega}^{\infty}\left(\Omega, \mathbb{R}^{2}, \beta\right)$ is a finite projective modular over $C_{\omega}^{\infty}\left(\Omega, \mathbb{R}^{2}, \beta\right)$. Given the derivations $\delta_{1}$ and $\delta_{2}$, a compatible connection $\nabla$ on $\mathscr{E}_{P}$ is a pair of linear maps $\nabla_{1}, \nabla_{2}: \mathscr{E}_{P} \rightarrow \mathscr{E}_{P}$ such that $\nabla_{j}(\xi u)=\nabla_{j}(\xi) u+\xi \delta_{j}(u)$ for $\xi \in \mathscr{E}_{P}$ and $u \in C_{\omega}^{\infty}\left(\Omega, \mathbb{R}^{2}, \beta\right)$. We can simply choose these maps to be $\nabla_{j}(\xi)=P \delta_{j}(\xi), j=1,2$. A simple calculation shows $\left(\nabla_{1} \nabla_{2}-\nabla_{2} \nabla_{1}\right) \xi=\left[P D_{1} P, P D_{2} P\right] \xi$. Therefore the curvature $\mathscr{C}=\nabla_{1} \nabla_{2}-\nabla_{2} \nabla_{1}$ is an endomorphism on $\mathscr{E}_{P}$, i.e. $\mathscr{C}(\xi u)=\mathscr{C}(\xi) u$ for $\xi \in \mathscr{E}_{P}$ and $u \in C_{\omega}^{\infty}\left(\Omega, \mathbb{R}^{2}, \beta\right)$. On the other hand, $P\left[\delta_{1}(P), \delta_{2}(P)\right] P=\left[P D_{1} P, P D_{2} P\right]=$ $\nabla_{1} \nabla_{2}-\nabla_{2} \nabla_{1}=\mathscr{C}$. Hence $\tau\left(P\left[\delta_{1}(P), \delta_{2}(P)\right]\right) / 2 \pi i=\tau(\mathscr{C}) / 2 \pi i$ if one extends, or, perhaps more appropriately, restricts $\tau$ to End $\mathscr{E}_{P}$. The main purpose of this paper is to identify the geometric invariant $\tau\left(P_{F}\left[\delta_{1}\left(P_{F}\right), \delta_{2}\left(P_{F}\right)\right]\right) / 2 \pi i$ with a topological invariant which is obviously an integer. To accomplish this, we need the pairing between the $K$-theory and the cyclic cohomology theory of the algebra of observables, and the Thom isomorphisms for $K$-groups and those for cyclic cohomology groups.

Recall that there is an operator $S$ for the cyclic cohomology theory such that for any algebra $\mathscr{A}, S: H_{\lambda}^{n}(\mathscr{A}) \rightarrow H_{\lambda}^{n+2}(\mathscr{A})$ is a group homomorphism [15]. Using this operator, one obtains two groups of inductive limits $H_{\lambda}^{\text {ev }}(\mathscr{A})=\underset{\mathrm{S}}{\lim _{\vec{S}}} H^{2 n}(\mathscr{A})$ and $H_{\lambda}^{\text {odd }}(\mathscr{A})=\underset{S}{\lim } H^{2 n+1}(\mathscr{A})$. There are pairings between $K_{0}(\mathscr{A})$ and $H_{\lambda}^{\text {ev }}(\mathscr{A})$ and between $K_{1}(\mathscr{A})$ and $H_{\lambda}^{\text {odd }}(\mathscr{A})$. That is, there are bilinear maps $\langle.,\rangle:. H_{\lambda}^{\text {ev }}(\mathscr{A}) \times$ $K_{0}(\mathscr{A}) \rightarrow \mathbb{R}$ and $\langle.,\rangle:. H_{\lambda}^{\text {odd }}(\mathscr{A}) \times K_{1}(\mathscr{A}) \rightarrow \mathbb{R}$. For the exact definition of these pairings, the reader is referred to [15]. As we have mentioned, the tri-linear functional $\psi$ defined by (1.11) is a cyclic 2-cocycle. Hence $\psi$ gives rise to a class $[\psi] \in H_{\lambda}^{\text {ev }}\left(C^{\infty}\left(\Omega, \mathbb{R}^{2}, \beta\right)\right)$. Thus $\langle[\psi],\rangle:. K_{0}\left(C^{\infty}\left(\Omega, \mathbb{R}^{2}, \beta\right)\right) \rightarrow \mathbb{R}$ is a group homomorphism. We will also consider $\langle[\psi]$, . $\rangle$ as a homomorphism from $K_{0}\left(C_{\omega}^{\infty}\left(\Omega, \mathbb{R}^{2}, \beta\right)\right)$ into $\mathbb{R}$. We will show that the range of this map is $\mathbb{Z}$. For a projection $P \in C_{\omega}^{\infty}\left(\Omega, \mathbb{R}^{2}, \beta\right),\langle[\psi],[P]\rangle=\tau\left(P\left[\delta_{1}(P), \delta_{2}(P)\right]\right) / 2 \pi i[15,108]$. In particular, the Hall conductivity can be expressed as

$$
\sigma_{H}=\left(e^{2} / 2 \pi \hbar\right)\left\langle[\psi],\left[P_{F}\right]\right\rangle .
$$

In [22], Kunz considered the Hall effect where the physical units are normalized so that $\beta=1$, and where the potential is relatively small, i.e. $\|Q\|_{\infty}<1 / 2$. It follows from general principles of analysis that, in this case, the spectrum of $H_{\omega}$ is contained in $B=\bigcup_{n=0}^{\infty} B_{n}$, where $B_{n}=\left[n+(1 / 2)-\|Q\|_{\infty}, n+(1 / 2)+\|Q\|_{\infty}\right]$. He showed that 
if $\lambda \notin B$, that is, if $\lambda$ belongs to a "natural gap" of the spectrum of the Hamiltonian, then the conductivity corresponding to $(-\infty, \lambda)$ is quantized and is given by the corresponding Landau band index. Using our notations, this amounts to computing $\left\langle[\psi],\left[P_{\lambda}\right]\right\rangle$ if $P_{\lambda}$ is the spectral projection of $H_{\omega}$ corresponding to $(-\infty, \lambda)$. He achieved this by approximating $Q$ by periodic potentials. But from the viewpoint of $K$-theory and cyclic cohomology theory, there is a much simpler approach. For $t \in[0,1]$, define $H_{\omega}^{t}=H_{0}+t Q_{\omega}$. The spectrum of every $H_{\omega}^{t}$ is also contained in $B$. The resolvent $R_{\omega}^{t}(z)=\left(z-H_{\omega}^{t}\right)^{-1}$ is a continuous function of $t$. Therefore, if $P_{\lambda}^{t}$ is the spectral projection of $H_{\omega}^{t}$ for the interval $(-\infty, \lambda)$, then $t \mapsto P_{\lambda}^{t}$ is a continuous function from $[0,1]$ onto $C_{\omega}^{\infty}\left(\Omega, \mathbb{R}^{2}, 1\right)$. Hence in $K_{0}\left(C_{\omega}^{\infty}\left(\Omega, \mathbb{R}^{2}, 1\right)\right),\left[P_{\lambda}\right]=\left[P_{\lambda}^{1}\right]=$ $\left[P_{\lambda}^{0}\right]$. Thus $\left\langle[\psi],\left[P_{\lambda}\right]\right\rangle=\left\langle[\psi],\left[P_{\lambda}^{0}\right]\right\rangle$. Since $H_{\omega}^{0}=H_{0}, P_{\lambda}^{0} \in \mathscr{K} \cap C_{\omega}^{\infty}\left(\Omega, \mathbb{R}^{2}, 1\right)=$ $\mathscr{K}_{\infty}=C^{\infty}$ (point, $\left.\mathbb{R}^{2}, 1\right)$, the class $\left[P_{\lambda}\right]$ in $K_{0}\left(C_{\omega}^{\infty}\left(\Omega, \mathbb{R}^{2}, 1\right)\right)$ belongs to the image of the inclusion map $l_{*}: K_{0}\left(\mathscr{K}_{\infty}\right) \rightarrow K_{0}\left(C^{\infty}\left(\Omega, \mathbb{R}^{2}, 1\right)\right)$. It is known that the pairing of $[\psi]$ with any element in $K_{0}\left(\mathscr{K}_{\infty}\right)$ is an integer. The main contribution of Kunz's work is, of course, the treatment of the case where the Fermi energy lies in the edge of one of the bands $B_{n}$, where the spectral projection is sufficiently localized.

Given a $C^{*}$-dynamical system $(A, \mathbb{R}, \alpha)$, there are two Thom isomorphisms $\phi_{\alpha, j}: K_{j}(A) \rightarrow K_{1-j}\left(A \times{ }_{\alpha} \mathbb{R}\right), j=0,1[13]$. For the algebra $C^{*}\left(\Omega, \mathbb{R}^{2}, \beta\right)=$ $\left[C(\Omega) \times{ }_{\theta} \mathbb{R}\right] \times{ }_{\gamma} \mathbb{R}$, the composition of the Thom isomorphisms $\phi_{\theta, 0}: K_{0}(C(\Omega)) \rightarrow$ $K_{1}\left(C(\Omega) \times{ }_{\theta} \mathbb{R}\right)$ and $\phi_{\gamma, 1}: K_{1}\left(C(\Omega) \times{ }_{\theta} \mathbb{R}\right) \rightarrow K_{0}\left(\left[C(\Omega) \times{ }_{\theta} \mathbb{R}\right] \times{ }_{\gamma} \mathbb{R}\right)$ provides an isomorphism

$$
\phi=\phi_{\gamma, 1}{ }^{\circ} \phi_{\theta, 0}: K_{0}(C(\Omega)) \rightarrow K_{0}\left(\left[C(\Omega) \times{ }_{\theta} \mathbb{R}\right] \times{ }_{\gamma} \mathbb{R}\right)=K_{0}\left(C^{*}\left(\Omega, \mathbb{R}^{2}, \beta\right)\right) .
$$

Because of Proposition 1.1 and because of the isomorphism $\pi_{\omega}$, for a.e. $\omega \in \Omega, \phi^{-1}$ can be considered as an isomorphism from $K_{0}\left(C_{\omega}^{\infty}\left(\Omega, \mathbb{R}^{2}, \beta\right)\right)$ onto $K_{0}(C(\Omega))$. The map $f \mapsto \int_{\Omega} f(\omega) d \mu(\omega)$ defines a 0-cocycle on $C(\Omega)$; its class in $H_{\lambda}^{\text {ev }}(C(\Omega))$ will be denoted by $[\mu]$. It is trivial that $\langle[\mu],[e]\rangle \in \mathbb{Z}$ for any $[e] \in K_{0}(C(\Omega))$. The following is the main result of the paper.

Theorem 3.3. For any $[P] \in K_{0}\left(C^{\infty}\left(\Omega, \mathbb{R}^{2}, \beta\right)\right)$, we have

$$
\langle[\psi],[P]\rangle=-\left\langle[\mu], \phi^{-1}[P]\right\rangle \in \mathbb{Z} .
$$

The proof of this theorem will be presented in the next section. Since the elements in $K_{0}(C(\Omega))$ can be identified as $\left[V_{1}\right]-\left[V_{2}\right]$, where $V_{1}$ and $V_{2}$ are vector bundles on $\Omega$, we can interpret the Hall conductivity as follows.

Corollary 3.4. If the Fermi energy $E_{F}$ belongs to a gap of the spectrum of $H_{\omega}$, then there are vector bundles $V^{+}$and $V^{-}$on $\Omega$ such that $\phi^{-1}\left[P_{F}\right]=\left[V^{+}\right]-\left[V^{-}\right]$and

$$
\sigma_{H}=\left(e^{2} / 2 \pi \hbar\right)\left(\operatorname{dim} V^{-}-\operatorname{dim} V^{+}\right) .
$$

At this point, it is necessary to compare our result with Bellissard's work on the same subject. In $[5,6,7]$, he considered the symmetric Hamiltonian

$$
H(\omega, \beta)=\left[(-i \partial / \partial x+\beta y / 2)^{2}+(-i \partial / \partial y-\beta x / 2)^{2}\right] / 2+Q_{\omega}(x, y)=W_{\beta} H_{\omega} W_{\beta}^{*},
$$

where $\left(W_{\beta} f\right)(x, y)=\exp (-i \beta x y / 2) f(x, y)$ for $f \in L^{2}\left(\mathbb{R}^{2}\right)$. Accordingly, the algebra of observables constructed there has the form $\mathscr{A}_{\omega}=W_{\beta} C_{\omega}^{*}\left(\Omega, \mathbb{R}^{2}, \beta\right) W_{\beta}^{*}$. That is, a 
function $a \in C_{c}\left(\Omega \times \mathbb{R}^{2}\right)$ represents an element

$$
\left(\tilde{\pi}_{\omega}(a) f\right)(x, y)=\int_{\mathbb{R}^{2}} a(\omega+(x, y), \xi, \eta) \exp (i \beta(y \xi-x \eta) / 2) f(x+\xi, y+\eta) d \xi d \eta
$$

in $\mathscr{A}_{\omega}$. To compute the Hall conductivity, he constructed a Fredholm module $(\mathscr{H}, G, F)$ with $\mathscr{H}=L^{2}\left(\mathbb{R}^{2}\right)_{+} \oplus L^{2}\left(\mathbb{R}^{2}\right)_{-}, G=\left[\begin{array}{rr}1 & 0 \\ 0 & -1\end{array}\right]$,

$$
F=\left[\begin{array}{cc}
0 & (x+i y) /|x+i y| \\
(x-i y) /|x+i y| & 0
\end{array}\right], \quad \text { and } \mathbf{A}=\left[\begin{array}{cc}
\pi_{\omega}(A) & 0 \\
0 & \pi_{\omega}(A)
\end{array}\right]
$$

for $A \in \mathscr{A}$. He then derived the formula

$$
\psi\left(A_{0}, A_{1}, A_{2}\right) / 2 \pi i=\int_{\Omega} \operatorname{Tr}_{s}\left(\mathbf{A}_{0}\left[F, \mathbf{A}_{1}\right]\left[F, \mathbf{A}_{2}\right]\right) d \mu
$$

where $\operatorname{Tr}_{s}(T)=\operatorname{Tr}(G F[F, T]) / 2$. Thus for a projection $P \in \mathscr{A}$,

$$
\tau\left(P\left[\delta_{1}(P), \delta_{2}(P)\right]\right) / 2 \pi i=\int_{\Omega} \operatorname{Tr}_{s}(\mathbf{P}[F, \mathbf{P}][F, \mathbf{P}]) d \mu .
$$

From this and a formula of Connes [15], it follows

$$
\tau\left(P\left[\delta_{1}(P), \delta_{2}(P)\right]\right) / 2 \pi i=\operatorname{Index}\left(F_{P}\right),
$$

where $F_{P}$ is the restriction of $\{\mathbf{P} F \mathbf{P}\}_{+,-}$to $\mathbf{P} \mathscr{H}$, which is a Fredholm operator. Thus Bellissard identified the Hall conductivity as an analytical index $\operatorname{Index}\left(F_{P}\right)$. The importance of the formula (3.7) is that it holds true for projections in the Sobolev space $H_{1}(\mathscr{A})$, i.e. for projections $P \in \mathscr{W}$ with finite localization length

$$
\tau\left(\left[\delta_{1}(P)\right]^{2}+\left[\delta_{2}(P)\right]^{2}\right)<+\infty .
$$

Therefore he was able to identify the Hall conductivity as an analytical index when the Fermi energy lies in a gap of extended states in the spectrum of the Hamiltonian.

By contrast, our formula $\langle[\psi],[P]\rangle=-\left\langle[\mu], \phi^{-1}[P]\right\rangle$ identifies the Hall conductivity as a topological index $-\left\langle[\mu], \phi^{-1}[P]\right\rangle$. This formula not only provides an alternate interpretation of the Hall conductivity but also has the following advantages. First, as it is the case for pseudo-differential operators, topological index is generally considered to be more readily calculable. (When $Q=0$, for example, it follow immediately from the construction of the Thom isomorphisms that $-\left\langle[\mu], \phi^{-1}\left[P_{F}\right]\right\rangle$ coincides with the Landau band index.) Second, it suggests that the $K$-theory class $\phi^{-1}\left[P_{F}\right]$ may contain more physical information of the quantum Hall effect than the conductivity $\sigma_{H}$. One sees from our formula, for example, that the Hall conductivity should not be interpreted as Landau band index unless $\phi^{-1}\left[P_{F}\right]$ lies in the subgroup $\mathbb{Z}[1]$ of $K_{0}(C(\Omega)$ ). It would be extremely interesting if one could come up with an example where $\phi^{-1}\left[P_{F}\right] \in K_{0}(C(\Omega)) \backslash \mathbb{Z}[1]$, or equivalently, $\left[P_{F}\right] \in K_{0}\left(C_{\omega}^{*}\left(\Omega, \mathbb{R}^{2}, \beta\right)\right) \backslash K_{0}(\mathscr{K})$. We would consider such a class as a nontrivial topological invariant of the quantum Hall effect. Given the fact that $K_{0}(C(\Omega))$ is generally quite larger for $\Omega$ with sufficiently complicated topology, this is not entirely inconceivable. But while our approach to the quantization of the Hall conductivity has its mathematical beauty, it also has the shortcoming that the Fermi energy is required to be in a gap of the 
spectrum of the Hamiltonian. With Bellissard's results for Sobolev projections in mind, one certainly would like to extend Theorem 3.4 to this class of projections. But that is the task of another paper. In the meantime, combining Bellissard's work and ours, we obtain the following analogue of the classical index theorem (analytical index $)=-($ topological index):

$$
\operatorname{Index}\left(F_{P}\right)=-\left\langle[\mu], \phi^{-1}[P]\right\rangle \quad P \in \operatorname{Proj} C_{\omega}^{\infty}\left(\Omega, \mathbb{R}^{2}, \beta\right) .
$$

\section{The Thom Isomorphisms and the Pairing}

As a first step in the proof of Theorem 3.3, let us examine how the two derivations $\delta_{1}$ and $\delta_{2}$ are constructed on $\left[C(\Omega) \times{ }_{\theta} \mathbb{R}\right] \times{ }_{\gamma} \mathbb{R}$ when one uses this iterative crossed product to represent $C^{*}\left(\Omega, \mathbb{R}^{2}, \beta\right)$. On the space $L^{2}\left(\Omega \times \mathbb{R}_{y}\right)$, where $C(\Omega) \times{ }_{\theta} \mathbb{R}$ lives, one defines the operator $\left(\widetilde{D}_{2} f\right)(\omega, y)=2 \pi i y f(\omega, y)$. Then $A \leftrightarrow\left[A, \widetilde{D}_{2}\right]=\widetilde{\delta}_{2}(A)$ extends to a closed derivation on $C(\Omega) \times{ }_{\theta} \mathbb{R}$. Since this derivation commutes with the automorphism group $\left\{\gamma_{s}: s \in \mathbb{R}\right\}$, it can be elevated to a derivation on $\left[C(\Omega) \times{ }_{\theta} \mathbb{R}\right] \times{ }_{\gamma} \mathbb{R}$ in the following way. For an element $\left.a \in C_{c}\left(\mathbb{R}, C(\Omega) \times{ }_{\theta} \mathbb{R}\right)\right)$, which is the collection of compactly supported, $C(\Omega) \times{ }_{\theta} \mathbb{R}$-valued continuous functions, such that $a(x)$ belongs to the domain of $\widetilde{\delta}_{2}$ for every $x$ and $x \mapsto \widetilde{\delta}_{2}(a(x))$ is continuous, simply define $\delta_{2}(a)(x)=\tilde{\delta}_{2}(a(x))$. One can easily check that $\delta_{2}$ extends to a closed derivation on $\left[C(\Omega) \times{ }_{\theta} \mathbb{R}\right] \times{ }_{\gamma} \mathbb{R}$ and that it is precisely the derivation denoted by the same symbol on $C^{*}\left(\Omega, \mathbb{R}^{2}, \beta\right)$ when these algebras are identified with each other. Similarly, $a \mapsto 2 \pi i x a\left(a \in C_{c}\left(\mathbb{R}, C(\Omega) \times{ }_{\theta} \mathbb{R}\right)\right)$ extends to the derivation $\delta_{1}$ on $\left[C(\Omega) \times{ }_{\theta} \mathbb{R}\right] \times{ }_{\gamma} \mathbb{R}$. The trace $\tau$ on $\left[C(\Omega) \times{ }_{\theta} \mathbb{R}\right] \times{ }_{\gamma} \mathbb{R}$ can be realized as follows. For a compactly supported, $C(\Omega) \times{ }_{\theta} \mathbb{R}$-valued continuous function $a(x)$, which represents an element $a$ in $\left[C(\Omega) \times{ }_{\theta} \mathbb{R}\right] \times{ }_{\gamma} \mathbb{R}$,

$$
\tau(a)=\tau_{1}(a(0)) \text {. }
$$

In order to prove Theorem 3.3, we need the Thom isomorphism for cyclic cohomology introduced recently by G. Elliott, T. Natsume and R. Nest [17]. Let us briefly recall the construction of this map. For a locally convex topological algebra $\mathscr{A}$, there is a universal differential graded algebra $(\Omega(\mathscr{A}), d)$ with $\boldsymbol{\Omega}(\mathscr{A})=\underset{n \geq 0}{\oplus} \boldsymbol{\Omega}_{n}(\mathscr{A})[15,17]$. (In this paper, we will only consider those $\mathscr{A}$ 's which are norm dense subalgebras of $C^{*}$-algebras.) Let $\mathscr{S} *(\mathbb{R})$ be the collection of smooth, rapidly decreasing function on $\mathbb{R}$ with convolution as its product. The differential graded algebra $\boldsymbol{\Omega}\left(\mathscr{S}^{*}(\mathbb{R})\right) / \oplus\left\{\boldsymbol{\Omega}_{n}\left(\mathscr{S}^{*}(\mathbb{R})\right): n \geqq 2\right\}$ will be denote by $\mathbf{E}$. Suppose that $\mathscr{A}$ is a Fréchet algebra with a smooth action $v$ of $\mathbb{R}$. Let $\left(\mathscr{A} \times{ }_{v} \mathbb{R}\right)^{\infty}$ denote the set of $\mathscr{A}$-valued smooth, rapidly decreasing function on $\mathbb{R}$ with the product structure $a * b(t)=\int_{\mathbb{R}} a(s) v_{s}(b(t-s)) d s$. (We will use the superscript $\infty$ to distinguish this smooth crossed product from the ordinary crossed product.) The space $\boldsymbol{\Omega}(\mathscr{A}) \otimes_{\nu} \mathbf{E}$ will be given a structure of locally convex differential graded algebra as follows [17].

(1) For $w \in \boldsymbol{\Omega}(\mathscr{A})$ and $f \in \mathbf{E}$,

$$
d(w \otimes f)=d w \otimes f+(-1)^{\operatorname{deg} w} w \otimes d f .
$$

(2) Define a left $\mathbf{E}_{0}$-module structure on $\boldsymbol{\Omega}(\mathscr{A}) \otimes \mathbf{E}_{0}$ as the one induced from the product structure of $\left(\boldsymbol{\Omega}(\mathscr{A})^{2} \times{ }_{v} \mathbb{R}\right)^{\infty}$. 
(3) Define a left $\mathbf{E}$-module structure on $\boldsymbol{\Omega}(\mathscr{A}) \otimes \mathbf{E}$ by the formulas $f(w \otimes g d h)=(f w \otimes g) d h, \quad d f(w \otimes g)=d(f w \otimes g)-f d(w \otimes g), \quad d f(w \otimes g d h)=0$.

(4) Define the product on $\boldsymbol{\Omega}(\mathscr{A}) \otimes \mathbf{E}$ by

$$
(w \otimes f)\left(w_{1} \otimes f_{1}\right)=w\left(f\left(w_{1} \otimes f_{1}\right)\right) .
$$

Given a closed continuous $n$-trace $T$ on $\boldsymbol{\Omega}(\mathscr{A})$, define an $n+1$-trace $\#_{\nu} T$ on $\boldsymbol{\Omega}(\mathscr{A}) \otimes{ }_{v} \mathbf{E}$ as follows. For $f \in \boldsymbol{\Omega}_{n}(\mathscr{A}) \otimes \mathscr{S}^{*}(\mathbb{R}) \otimes \mathscr{S}^{*}(\mathbb{R}) \subset \boldsymbol{\Omega}_{n}(\mathscr{A}) \otimes \mathbf{E}_{1}$,

$$
\#_{\nu} T(f)=2 \pi i \int_{\mathbb{R}}\left(\int_{0}^{t} T\left(v_{\lambda}(f(-t, t))\right) d \lambda\right) d t .
$$

For other $f, \#_{v} T(f)=0$. It was shown in [9] that $\#_{v}$ induces isomorphisms

$$
\begin{aligned}
H_{\lambda}^{\mathrm{ev}}(\mathscr{A}) & \rightarrow H_{\lambda}^{\text {odd }}\left(\left(\mathscr{A} \times{ }_{v} \mathbb{R}\right)^{\infty}\right), \\
H_{\lambda}^{\text {odd }}(\mathscr{A}) & \rightarrow H^{\text {ev }}\left(\left(\mathscr{A} \times{ }_{v} \mathbb{R}\right)^{\infty}\right) .
\end{aligned}
$$

Given a smooth action $v$ on a Fréchet algebra $\mathscr{A}$, there is a dual action $\hat{v}$ on $\left(\mathscr{A} \times{ }_{v} \mathbb{R}\right)^{\infty}$. Indeed if $f \in\left(\mathscr{A} \times{ }_{v} \mathbb{R}\right)^{\infty}$ is regarded as an $\mathscr{A}$-valued function, then $\left(\hat{v}_{s} f\right)(t)=e^{2 \pi i s} f(t)[15,17]$. It was shown in [17] that there is a natural isomorphism $T_{v}: \mathscr{A} \otimes \mathscr{K}^{\infty} \rightarrow\left[\left(\mathscr{A} \times{ }_{v} \mathbb{R}\right)^{\infty} \times{ }_{v} \mathbb{R}\right]^{\infty}$, where $\mathscr{K}^{\infty}$ is the collection of Hilbert-Schmidt operators on $L^{2}(\mathbb{R})$ with kernels in $\mathscr{S}\left(\mathbb{R}^{2}\right)$. There is a map $r: \mathscr{A} \rightarrow \mathscr{A} \otimes \mathscr{K}^{\infty}$ such that $r(a)=a \otimes e_{0}$, where $e_{0}$ is the projection corresponding to the $0^{\text {th }}$ Hermite function [17]. These maps induce an isomorphism $T_{v *}: K_{1}\left(\mathscr{A} \otimes \mathscr{K}^{\infty}\right) \rightarrow$ $K_{1}\left(\left[\left(\mathscr{A} \times{ }_{v} \mathbb{R}\right)^{\infty} \times \mathbb{R} \mathbb{R}\right]^{\infty}\right)$ and a homomorphism $r_{*}: K_{1}(\mathscr{A}) \rightarrow K_{1}\left(\mathscr{A} \otimes \mathscr{K}^{\infty}\right)$. Hence the map $T_{v} r: \mathscr{A} \rightarrow\left[\left(\mathscr{A} \times{ }_{v} \mathbb{R}\right)^{\infty} \times{ }_{v} \mathbb{R}\right]^{\infty}$ induces an homomorphism $\left(T_{v} r\right)_{*}=T_{v *} r_{*}$ : $K_{1}(\mathscr{A}) \rightarrow K_{1}\left(\left[\left(\mathscr{A} \times{ }_{v} \mathbb{R}\right)^{\infty} \times{ }_{\hat{v}} \mathbb{R}\right]^{\infty}\right)$. These maps also induce homomorphisms between cyclic cohomologies: $T_{v}^{*}: H_{\lambda}^{*}\left(\left[\left(\mathscr{A} \times{ }_{v} \mathbb{R}\right)^{\infty} \times{ }_{v} \mathbb{R}\right]^{\infty}\right) \rightarrow H_{\lambda}^{*}\left(\mathscr{A} \otimes \mathscr{K}^{\infty}\right)$ and $r^{*}: H_{\lambda}^{*}\left(\mathscr{A} \otimes \mathscr{K}^{\infty}\right) \rightarrow H_{\lambda}^{*}(\mathscr{A})$.

Lemma 4.1. Let $\Lambda$ be an odd cyclic cocycle on a Fréchet algebra $\mathscr{A}$ which admits a smooth action $v$ of $\mathbb{R}$ and let $[\Lambda]$ be the class of $\Lambda$ in $H_{\lambda}^{\text {odd }}(\mathscr{A})$. Then for any $v \in K_{1}(\mathscr{A})$,

$$
\left\langle \#_{v} \#_{v}[\Lambda], T_{v *} r_{*} v\right\rangle=\langle[\Lambda], v\rangle \text {. }
$$

Proof. By the definition of the pairing between $H_{\lambda}^{\text {odd }}$ and $K_{1}$, we have $\left\langle \#_{\nu} \#_{\nu}[\Lambda]\right.$, $\left.T_{v *} r_{*} v\right\rangle=\left\langle r^{*} T_{v}^{*} \#_{v} \#_{v}[\Lambda], v\right\rangle$. According to the proofs of Theorems 4.2 and 5.2 of $[17], r^{*} T_{v}^{*} \#_{v} \#_{v}[\Lambda]=[S \Lambda]=[\Lambda]$ in $H_{\lambda}^{\text {odd }}$. Therefore $\left\langle \#_{v} \#_{v}[\Lambda], T_{v *} r_{*} v\right\rangle=$ $\langle[\Lambda], v\rangle$.

Let $C^{\infty}(\Omega)$ be the collection of functions $f \in C(\Omega)$ such that $(x, y) \mapsto f \circ \varphi_{x, y}$ is a $C(\Omega)$-valued $C^{\infty}$ function on $\mathbb{R}^{2}$. Then the group $\theta_{t}=\varphi_{0, t}$ acts on $C^{\infty}(\Omega)$ smoothly. Hence we can form the smooth crossed product $\left(C^{\infty}(\Omega) \times{ }_{\theta} \mathbb{R}\right)^{\infty}$. If $\mu$ denotes the 0 -cocycle on $C^{\infty}(\Omega)$ of integration against the ergodic measure $d \mu$, then $\#_{\theta} \mu$ is a 1-cocycle on $\left(C^{\infty}(\Omega) \times{ }_{\theta} \mathbb{R}\right)^{\infty}$. The automorphism group $\left\{\gamma_{s}: s \in \mathbb{R}\right\}$ of $C(\Omega) \times{ }_{\theta} \mathbb{R}$ defined in Sect. 2 acts smoothly on the subalgebra $\left(C^{\infty}(\Omega) \times{ }_{\theta} \mathbb{R}\right)^{\infty}$. Thus we have a 2 -cocycle $\#_{\gamma} \#_{\theta} \mu$ on the smooth crossed product $\left[\left(C^{\infty}(\Omega) \times{ }_{\theta} \mathbb{R}\right)^{\infty} \times{ }_{\gamma} \mathbb{R}\right]^{\infty}$. When $\Omega$ is a single point, this cocycle was computed in [17]. We will do the same computation for nontrivial $\Omega$ 's. But as was the case in [17], this computation is no more than a chasing of the definition of the operator \#, the constructions of 
$\left(C^{\infty}(\Omega) \times{ }_{\theta} \mathbb{R}\right)^{\infty}$ and $\left[\left(C^{\infty}(\Omega) \times{ }_{\theta} \mathbb{R}\right)^{\infty} \times{ }_{\gamma} \mathbb{R}\right]^{\infty}$, and the traces and derivations thereon.

Lemma 4.2. (i) For any $b_{0}, b_{1} \in\left(C^{\infty}(\Omega) \times{ }_{\theta} \mathbb{R}\right)^{\infty}$,

$$
\#{ }_{\theta} \mu\left(b_{0}, b_{1}\right)=\tau_{1}\left(b_{0} \tilde{\delta}_{2}\left(b_{1}\right)\right) \text {, }
$$

where $\tau_{1}$ is the trace defined by (2.3) and $\tilde{\delta}_{2}$ the derivation defined in the first paragraph of this section.

(ii) For any $a_{0}, a_{1}, a_{2} \in\left[\left(C^{\infty}(\Omega) \times{ }_{\theta} \mathbb{R}\right)^{\infty} \times{ }_{\gamma} \mathbb{R}\right]^{\infty}$,

$$
\#_{\gamma} \#_{\theta} \mu\left(a_{0}, a_{1}, a_{2}\right)=\tau\left(a_{0}\left[\delta_{2}\left(a_{1}\right) \delta_{1}\left(a_{2}\right)-\delta_{1}\left(a_{1}\right) \delta_{2}\left(a_{2}\right)\right]\right)=-\psi\left(a_{0}, a_{1}, a_{2}\right) .
$$

Proof: (i) We start with the graded differential algebra $\left(\Omega\left(C^{\infty}(\Omega)\right) \otimes_{\theta} \mathbf{E}, d\right)$. Because of the linearity and continuity of $\#_{\theta} \mu$ on this algebra, it suffices to evaluate the 1-cocycle at elements $b_{0} d b_{1}$, where $b_{j}=\left(u_{j} \otimes f_{j}\right)$ with $u_{j} \in C^{\infty}(\Omega)$ and $f_{j} \in \mathscr{S}^{*}(\mathbb{R})$, $j=0,1$. $\left(u_{j} \otimes f_{j}\right.$ represents the element $u_{j}(\omega) f_{j}(y)$ in $\left(C^{\infty}(\Omega) \times{ }_{\theta} \mathbb{R}\right)^{\infty}$.) By the definitions (1),...,(4), we have $b_{0} d b_{1}=\left(u_{0} \otimes f_{0}\right) d\left(u_{1} \otimes f_{1}\right)=\left(u_{0} \otimes f_{0}\right)\left(d u_{1} \otimes f_{1}+u_{1} \otimes d f_{1}\right)=$ $u_{0}\left(f_{0}\left(d u_{1} \otimes f_{1}\right)\right)+u_{0}\left(f_{0}\left(u_{1} \otimes d f_{1}\right)\right)$. Since $u_{0}\left(f_{0}\left(d u_{1} \otimes f_{1}\right)\right) \in \boldsymbol{\Omega}_{1}\left(C^{\infty}(\Omega)\right) \otimes \mathbf{E}_{0}$ and, as an $\boldsymbol{\Omega}_{0}\left(C^{\infty}(\Omega)\right)$-valued function on $\mathbb{R}^{2}, u_{0}\left(f_{0}\left(u_{1} \otimes d f_{1}\right)\right)(s, t)=u_{0} \theta_{s}\left(u_{1}\right) f_{0}(s) f_{1}(t)$, by $(4.1)$,

$$
\begin{aligned}
\#_{\theta} \mu\left(b_{0}, b_{1}\right) & =2 \pi i \int_{\mathbb{B}}^{t} \int_{0}^{t} \int_{\Omega} \theta_{\lambda}\left(u_{0} \theta_{-t}\left(u_{1}\right) f_{0}(-t) f_{1}(t)\right) d \mu d \lambda d t \\
& =2 \pi i \int_{\Omega} \int_{\mathbb{R}} t u_{0}(\omega) \theta_{-t}\left(u_{1}(\omega)\right) f_{0}(-t) f_{1}(t) d t d \mu(\omega) \\
& =-2 \pi i \int_{\Omega} \int_{\mathbb{R}} t u_{0}(\omega) f_{0}(t) \theta_{t}\left(u_{1}(\omega)\right) f_{1}(-t) d t d \mu(\omega) \\
& =-\tau_{1}\left(\widetilde{\delta}_{2}\left(b_{0}\right) b_{1}\right)=\tau_{1}\left(b_{0} \widetilde{\delta}_{2}\left(b_{1}\right)\right) .
\end{aligned}
$$

(ii) Once again, it suffices to evaluate $\#_{\gamma} \#_{\theta} \mu$ at $a_{0} d a_{1} d a_{2}$, where $a_{j}=v_{j} \otimes g_{j}$ with $v_{j} \in\left(C^{\infty}(\Omega) \times{ }_{\theta} \mathbb{R}\right)^{\infty}$ and $g_{j} \in \mathscr{S}^{*}(\mathbb{R}), j=0,1,2$. By the definition of the product on $\Omega\left(\left(C^{\infty}(\Omega) \times{ }_{\theta} \mathbb{R}\right)^{\infty}\right) \otimes_{\gamma} \mathbf{E}$, we have

$$
\begin{aligned}
\left(v_{0} \otimes g_{0}\right) d\left(v_{1} \otimes g_{1}\right) d\left(v_{2} \otimes g_{2}\right)= & \int_{\mathbb{R}} v_{0} d\left(\gamma_{r}\left(v_{1}\right) \gamma_{s}\left(v_{2}\right)\right) g_{0}(r) g_{1}(s-r) d r g_{2}(t) \\
& -\int_{\mathbb{R}} v_{0} \gamma_{s}\left(v_{1} d\left(\gamma_{r}\left(v_{2}\right)\right)\right) g_{0}(s) g_{1}(r) g_{2}(t-r) d r+A,
\end{aligned}
$$

where the first two terms are functions in $\boldsymbol{\Omega}_{1}\left(\left(C^{\infty}(\Omega) \times{ }_{\theta} \mathbb{R}\right)^{\infty}\right) \otimes \mathscr{S}^{*}(\mathbb{R}) \otimes \mathscr{S}^{*}(\mathbb{R})$ and $A$ does not belong to this space. Applying (4.1) to the first two terms and using the definitions of $\tau_{1}, \widetilde{\delta}_{2}, \tau, \delta_{1}, \delta_{2}$, we have

$$
\begin{aligned}
\#_{\gamma} \#_{\theta} \mu\left(a_{0}, a_{1}, a_{2}\right) & =-\tau\left(\delta_{1}\left(a_{0} \delta_{2}\left(a_{1}\right)\right) a_{2}\right)-\tau\left(\delta_{1}\left(a_{0} a_{1}\right) \delta_{2}\left(a_{2}\right)\right)+\tau\left(\delta_{1}\left(a_{0}\right) a_{1} \delta_{2}\left(a_{2}\right)\right) \\
& =\tau\left(a_{0} \delta_{2}\left(a_{1}\right) \delta_{1}\left(a_{2}\right)\right)-\tau\left(a_{0} \delta_{1}\left(a_{1}\right) \delta_{2}\left(a_{2}\right)\right)=-\psi\left(a_{0}, a_{1}, a_{2}\right) .
\end{aligned}
$$

For a nonunital algebra $\mathscr{A}$, let $\tilde{\mathscr{A}}$ denote the algebra obtained from $\mathscr{A}$ by adjoining an identity. For a complex algebra $\mathscr{A}$, in the pairing $\langle.,$.$\rangle :$ $H^{\text {odd }}(\mathscr{A}) \times K_{1}(\mathscr{A}) \rightarrow \mathbb{R}$ defined in [15], $K_{1}(\mathscr{A})$ is the algebraic $K_{1}$-group of $\mathscr{A}$. The algebraic $K_{1}(\mathscr{A})$ is defined as follows $[14,15]$. Let $G L_{n}(\tilde{\mathscr{A}})$ denote the collection of invertible elements in $\tilde{\mathscr{A}} \otimes M_{n}$, where $M_{n}$ is the collection of $n \times n$ matrices. Let $G L_{\infty}(\tilde{\mathscr{A}})$ denote the inductive limit of $G L_{n}(\tilde{\mathscr{A}})$ in the obvious way. If one 
denotes the subgroup of $G L_{\infty}(\tilde{\mathscr{A}})$ generated by commutators by $G L_{\infty}^{c}(\tilde{\mathscr{A}})$, then the algebraic $K_{1}(\mathscr{A})=K_{1}^{a}(\mathscr{A})$ is defined to be $G L_{\infty}(\tilde{\mathscr{A}}) / G L_{\infty}^{c}(\tilde{\mathscr{A}})$. If $\mathscr{A}$ is a Fréchet algebra and $\Lambda$ is an odd cyclic cocycle on $\mathscr{A}$ which is continuous with respect to the Fréchet topology, then it is possible to pair the class of $\Lambda$ in $H^{\text {odd }}(\mathscr{A})$ with a certain topological $K_{1}$-group of $\mathscr{A}$. We will make this statement precise for certain subalgebras of $C^{*}$-algebras.

Let $B$ be a $C^{*}$-algebra satisfying the following conditions:

(i) $B$ has a trace $\tau_{B}$ with a dense domain and the property that $\left|\tau_{B}(a b)\right| \leqq$ $\|a\| \tau_{B}\left(\left(b^{*} b\right)^{1 / 2}\right)$ for any $a \in B$ and any $\tau_{B}$-traces class element $b \in B$.

(ii) $B$ is equipped with closed, densely defined derivations $\partial_{1}, \ldots, \partial_{p}$ which commute with each other.

Let $\mathscr{B}$ be the collection of $a \in B$ such that for any nonnegative integers $n_{1}, \ldots, n_{p}$, Define

(iii) $a_{n_{1}, \ldots, n_{p}}=\partial_{1}^{n_{1}} \cdots \partial_{p}^{n_{p}}(a) \in B$ and $\tau_{B}\left(a_{n_{1}, \ldots, n_{p}}^{*} a_{n_{1}, \ldots, n_{p}}\right)<\infty$.

(iv) $\|a\|_{n_{1}, \ldots, n_{p}}=\left\|a_{n_{1}, \ldots, n_{p}}\right\|+\left(\tau_{B}\left(a_{n_{1}, \ldots, n_{p}}^{*} a_{n_{1}, \ldots, n_{p}}\right)\right)^{1 / 2}$ for $a \in \mathscr{B}$.

With these norms, $\mathscr{B}$ becomes a Fréchet algebra. The algebra $\mathscr{B}$ is closed under holomorphic functional calculus [13]. Thus each $G L_{n}(\widetilde{\mathscr{B}})$ contains a subgroup $\exp _{n}(\tilde{\mathscr{B}})$ consisting of $\exp \left(a_{1}\right) \cdots \exp \left(a_{m}\right)$ with $a_{1}, \ldots, a_{m} \in \tilde{\mathscr{B}} \otimes M_{n}$. Let $\exp _{\infty}(\widetilde{\mathscr{B}})$ be the inductive limit of $\exp _{n}(\mathscr{\mathscr { B }})$. For an algebra $\mathscr{B}$ satisfying (i), (ii) and (iii), we use the symbol $K_{1}(\mathscr{B})$ to denote $G L_{\infty}(\tilde{\mathscr{B}}) / \exp _{\infty}(\tilde{\mathscr{B}})$ rather than the usual algebraic $K_{1}$-group $G L_{\infty}(\tilde{\mathscr{B}}) / G L_{\infty}^{c}(\widetilde{\mathscr{B}})$, which will now be denoted by $K_{1}^{a}(\mathscr{B})$. (For Fréchet algebras $\mathscr{A}$ which does not satisfy (i), (ii) and (iii) and which is not a $C^{*}$-algebra, $K_{1}(\mathscr{A})$ will still denote the algebraic $K_{1}$-group.) Since $\exp _{\infty}(\tilde{\mathscr{B}}) \supset G L_{\infty}^{c}(\tilde{\mathscr{B}})$, there is a natural surjective homomorphism $\mathscr{I}: K_{1}^{a}(\mathscr{B}) \rightarrow K_{1}(\mathscr{B})$. If $\mathscr{B}$ is a dense subalgebra of $B$, then the inclusion map $l: \mathscr{B} \rightarrow B$ induces an isomorphism $\imath_{*}: K_{1}(\mathscr{B}) \rightarrow K_{1}(B)$ [15].

Let $\Lambda$ be a cyclic $(2 k-1)$-cocycle on $\mathscr{B}$ which is continuous with respect to the Fréchet topology (iv). We will now show that there is a pairing of the class of $\Lambda$ in $\left.H_{\lambda}^{\text {odd }} \mathscr{B}\right)$ with $K_{1}(\mathscr{B})$ such that for any $u \in K_{1}^{a}(\mathscr{B})$, the pairing of $[\Lambda]$ with $\mathscr{I} u$ $\left(\in K_{1}(\mathscr{B})\right)$ equals $\langle[\Lambda], u\rangle$. Because of the pairing between $H_{\lambda}^{\text {odd }}(\mathscr{B})$ and $K_{1}^{a}(\mathscr{B})$, it suffices to show that the $\langle[\Lambda], v\rangle=0$ for any $v \in \exp _{\infty}(\tilde{\mathscr{B}}) / G L_{\infty}^{c}(\tilde{\mathscr{B}})=\operatorname{ker} \mathscr{I}$. Furthermore, because of the linearity, it suffices to show that $\langle[\Lambda]$, $[\exp (a)]\rangle=0$ for any $a \in \tilde{\mathscr{B}} \otimes M_{n}$. Recall that to evaluate this pairing, one first extends $\Lambda$ to $\widetilde{\mathscr{B}}$ such that $\Lambda\left(1, a_{1}, \ldots, a_{2 k-1}\right)=0$ [15]. It is a routine calculation to verify that $\Lambda$ is a cyclic cocycle on $\widetilde{\mathscr{B}}$. For any $w \in G L_{n}(\tilde{\mathscr{B}}),\langle[\Lambda],[w]\rangle=c_{k}\left(\Lambda \# \operatorname{tr}_{n}\right) \times$ $\left(w^{-1}-1, w-1, \ldots, w^{-1}-1, w-1\right)$, where $c_{k}$ is a coefficient depending only on $k$ [15]. Since $t \mapsto \exp (t a)$ is a function differentiable with respect to the topology (iv), and since $\Lambda$ is continuous with respect to this topology, one can repeat the argument in $[15, \mathrm{pp} .109,110]$ to show that $\langle[\Lambda],[\exp (a)]\rangle=0$. But there is a simpler way to do this. By the linearity of the pairing, we have, for any natural number $p,\langle[\Lambda],[\exp (a)]\rangle=p\langle[\Lambda],[\exp (a / p)]\rangle=c_{k}\left(\Lambda \# \operatorname{tr}_{n}\right)([\exp (-a / p)-1] /$ $\left.\left[(1 / p)^{1 / 2 k}\right], \ldots,[\exp (a / p)-1] /\left[(1 / p)^{1 / 2 k}\right]\right)$. Since $\lim [\exp (a / p)-1] /(1 / p)=a$ in the topology (iv), we have, for $k \geqq 1, \lim _{p \rightarrow \infty}\left[\exp (a / p)^{p \rightarrow \infty}-1\right] /\left[(1 / p)^{1 / 2 k}\right]=0$. Thus, by the continuity of $\Lambda,\langle[\Lambda],[\exp (a)]\rangle=0$. Hence for any cyclic odd-cocycle $\Lambda$ on $\mathscr{B}$ which is continuous with respect to the topology (iv), there is a homomorphism 
$\langle[\Lambda],\rangle:. K_{1}(\mathscr{B}) \rightarrow \mathbb{R}$ such that $\langle[\Lambda], \mathscr{I} u\rangle=\langle[\Lambda], u\rangle$ for $u \in K_{1}^{a}(\mathscr{B})$. In what follows, the pairing between the $K_{1}$-group of a holomorphically closed subalgebra of a $C^{*}$-algebra equipped with a Fréchet topology (iv) and a continuous cyclic odd-cocycle will be understood in this sense.

Let $\left[C(\Omega) \times{ }_{\theta} \mathbb{R}\right]_{\infty}$ be the collection of elements $A \in C(\Omega) \times{ }_{\theta} \mathbb{R}$ such that for any nonnegative integers $m, n, \widetilde{\delta}_{2}^{m}\left(\delta_{\gamma}^{n}(A)\right) \in C(\Omega) \times{ }_{\theta} \mathbb{R}$ and $\tau_{1}\left(\left[\widetilde{\delta}_{2}^{m}\left(\delta_{\gamma}^{n}(A)\right)\right]^{*} \tilde{\delta}_{2}^{m}\left(\delta_{\gamma}^{n}(A)\right)\right)<\infty$. Here, $\delta_{\gamma}$ is the derivation induced by the automorphism group $\left\{\gamma_{s}: s \in \mathbb{R}\right\}$. Obviously (i)-(iv) are satisfied by $\mathscr{B}=\left[C(\Omega) \times{ }_{\theta} \mathbb{R}\right]_{\infty}$. Thus we have an isomorphism $l_{*}$ : $K_{1}\left(\left[C(\Omega) \times{ }_{\theta} \mathbb{R}\right]_{\infty}\right) \rightarrow K_{1}\left(C(\Omega) \times{ }_{\theta} \mathbb{R}\right)$. Therefore the Thom isomorphism $\phi_{\theta, 0}$ : $K_{0}(C(\Omega)) \rightarrow K_{1}\left(C(\Omega) \times{ }_{\theta} \mathbb{R}\right)$ can be regarded as an isomorphism $\phi_{\theta, 0}: K_{0}\left(C^{\infty}(\Omega)\right) \rightarrow$ $K_{1}\left(\left[C(\Omega) \times{ }_{\theta} \mathbb{R}\right]_{\infty}\right)$. On the algebra $\left[C(\Omega) \times{ }_{\theta} \mathbb{R}\right]_{\infty}$, define a cyclic 1 -cocycle

$$
\Lambda_{\theta}\left(a_{0}, a_{1}\right)=\tau_{1}\left(a_{0} \widetilde{\delta}_{2}\left(a_{1}\right)\right), \quad a_{0}, a_{1} \in\left[C(\Omega) \times{ }_{\theta} \mathbb{R}\right]_{\infty} .
$$

Obviously, $\Lambda_{\theta}$ is a continuous extention of the cyclic cocycle $\#_{\theta} \mu$ on $\left(C^{\infty}(\Omega) \times{ }_{\theta} \mathbb{R}\right)^{\infty}$ to $\left[C(\Omega) \times{ }_{\theta} \mathbb{R}\right]_{\infty}$.

Lemma 4.3. For any $u \in K_{1}\left(\left[C(\Omega) \times{ }_{\theta} \mathbb{R}\right]_{\infty}\right)$,

$$
\left\langle\left[\Lambda_{\theta}\right], u\right\rangle=\left\langle[\mu], \phi_{\theta, 0}^{-1} u\right\rangle .
$$

Proof. It was shown in $\left[17\right.$, Sect. 6.1] that $u=\phi_{\theta, 0}[e]$, where $[e] \in K_{0}\left(C^{\infty}(\Omega)\right)$ and $\phi_{\theta, 0}[e]$ is represented by a class in $K_{1}\left(\left(C^{\infty}(\Omega) \times{ }_{\theta} \mathbb{R}\right)^{\infty}\right)$. Therefore $\left\langle\left[\Lambda_{\theta}\right], u\right\rangle=$ $\left\langle\left[\Lambda_{\theta}\right], \phi_{\theta, 0}[e]\right\rangle=\left\langle \#_{\theta}[\mu], \phi_{\theta, 0}[e]\right\rangle \quad[15, \mathrm{p} .109] . \quad$ By $\quad[17$, Theorem 6.2], $\left\langle \#_{\theta}[\mu], \phi_{\theta, 0}[e]\right\rangle=\langle[\mu],[e]\rangle=\left\langle[\mu], \phi_{\theta, 0}^{-1} u\right\rangle$.

Since the action of $\left\{\gamma_{s}: s \in \mathbb{R}\right\}$ on $\left[C(\Omega) \times{ }_{\theta} \mathbb{R}\right]_{\infty}$ is smooth, we can define the smooth crossed product

$$
\left(\left[C(\Omega) \times{ }_{\theta} \mathbb{R}\right]_{\infty} \times{ }_{\gamma} \mathbb{R}\right)^{\infty} .
$$

Accordingly, one obtains a cyclic 2-cocycle $\#_{\gamma} \Lambda_{\theta}$ on this algebra. By the definition of the \# operator, $\#_{\gamma} \Lambda_{\theta}$ is a continuous extension of $\#_{\gamma} \#_{\theta} \mu$ to $\left(\left[C(\Omega) \times{ }_{\theta} \mathbb{R}\right]_{\infty} \times \mathbb{R}\right)^{\infty}$. Because $-\psi$ is the continuous extension of $\#_{\gamma} \#_{\theta} \mu$ to $C^{\infty}\left(\Omega, \mathbb{R}^{2}, \beta\right)$, which contains $\left(\left[C(\Omega) \times{ }_{\theta} \mathbb{R}\right]_{\infty} \times{ }_{\gamma} \mathbb{R}\right)^{\infty}$, we have

$$
\#_{\gamma} \Lambda_{\theta}\left(a_{0}, a_{1}, a_{2}\right)=-\psi\left(a_{0}, a_{1}, a_{2}\right) .
$$

Let $\{\hat{\gamma}: s \in \mathbb{R}\}$ be the automorphism group on $\left[C(\Omega) \times{ }_{\theta} \mathbb{R}\right] \times{ }_{\gamma} \mathbb{R}=C^{*}\left(\Omega, \mathbb{R}^{2}, \beta\right)$. dual to the action of $\left\{\gamma_{s}: s \in \mathbb{R}\right\}$. The trace $\tau$ induces a trace $\hat{\tau}$ the algebra $C^{*}\left(\Omega, \mathbb{R}^{2}, \beta\right) \times{ }_{\hat{\gamma}} \mathbb{R}=\left(\left[C(\Omega) \times{ }_{\theta} \mathbb{R}\right] \times{ }_{\gamma} \mathbb{R}\right) \times{ }_{\hat{\gamma}} \mathbb{R}$ in the usual way. That is, for an $a \in C_{c}\left(\mathbb{R}, C^{*}\left(\Omega, \mathbb{R}^{2}, \beta\right)\right)$ representing an element in the triple crossed product, $\hat{\tau}(a)=\tau(a(0))$. Since $\delta_{1}$ and $\delta_{2}$ on $C^{*}\left(\Omega, \mathbb{R}^{2}, \beta\right)$ commute with the action of the automorphism group $\left\{\hat{\gamma}_{s}: s \in \mathbb{R}\right\}$, these derivations naturally induce derivations $\hat{\delta}_{1}$ and $\hat{\delta}_{2}$ on $C^{*}\left(\Omega, \mathbb{R}^{2}, \beta\right) \times{ }_{j} \mathbb{R}$. Indeed for an $a \in C_{c}\left(\mathbb{R}, C^{*}\left(\Omega, \mathbb{R}^{2}, \beta\right)\right)$, we have $\left(\hat{\delta}_{j}(a)\right)(t)=$ $\delta_{j}(a(t))$, if this function also represents an element in the triple crossed product, $j=1,2$. On this algebra, we define a third derivation $\left(\hat{\delta}_{3}(a)\right)(t)=2 \pi i t a(t)$ if the $C^{*}\left(\Omega, \mathbb{R}^{2}, \beta\right)$-valued functions $a(t)$ and $2 \pi i t a(t)$ represent elements in $C^{*}\left(\Omega, \mathbb{R}^{2}, \beta\right) \times \mathbb{R}$. It is obvious that the derivations $\hat{\delta}_{1}, \hat{\delta}_{2}$ and $\hat{\delta}_{3}$ commute. Let $\left[C^{*}\left(\Omega, \mathbb{R}^{2}, \beta\right) \times \mathbb{R}_{\mathbb{R}}\right]_{\infty}$ denote the collection of elements $A$ such that for any nonnegative integers $m, n, k, A_{n, m, k}=\hat{\delta}_{1}^{n} \hat{\delta}_{2}^{m} \hat{\delta}_{3}^{k}(A) \in C^{*}\left(\Omega, \mathbb{R}^{2}, \beta\right) \times \mathbb{R}_{\mathbb{R}}$ and $\hat{\tau}\left(A_{n, m, k}^{*} A_{n, m, k}\right)<\infty$. This algebra 
again satisfies (i)-(iv). Since $\left[C^{*}\left(\Omega, \mathbb{R}^{2}, \beta\right) \times{ }_{\hat{\gamma}} \mathbb{R}\right]_{\infty}$ is obviously dense in $C^{*}\left(\Omega, \mathbb{R}^{2}, \beta\right) \times$ ${ }_{\hat{\gamma}} \mathbb{R}$, we have an isomorphism

$$
l_{*}: K_{1}\left(\left[C^{*}\left(\Omega, \mathbb{R}^{2}, \beta\right) \times, \mathbb{R}\right]_{\infty}\right) \rightarrow K_{1}\left(C^{*}\left(\Omega, \mathbb{R}^{2}, \beta\right) \times{ }_{\hat{\gamma}} \mathbb{R}\right) .
$$

Since $C^{\infty}\left(\Omega, \mathbb{R}^{2}, \beta\right)$ is smooth under the action of $\left\{\hat{\gamma}_{s}: s \in \mathbb{R}\right\}$, one has the smooth crossed product $\left(C^{\infty}\left(\Omega, \mathbb{R}^{2}, \beta\right) \times{ }_{\hat{y}} \mathbb{R}\right)^{\infty}$. By (4.1) and by calculations similar to those in the proof of Lemma 4.2 , the cyclic 3-cocycle \# $\psi$ on $\left(C^{\infty}\left(\Omega, \mathbb{R}^{2}, \beta\right) \times \mathbb{R}\right)^{\infty}$ extends continuously to a cyclic 3-cocycle $\Psi$ on $\left[C^{*}\left(\Omega, \mathbb{R}^{2}, \beta\right) \times \mathbb{P}_{\hat{\gamma}}\right]_{\infty}$. Indeed if the calculation in the proof of Lemma 4.2(b) is carried out one step further, one finds that $\#_{\hat{\gamma}} \psi\left(a_{0}, a_{1}, a_{2}, a_{3}\right)$ can be expressed in terms of the $\hat{\tau}$-trace of a finite, linear and multiplicative combination of $a_{0}$ and derivations of $a_{1}, a_{2}$ and $a_{3}$. Hence we have a closed continuous cyclic 3 -cocycle $\Psi$ on $\left[C^{*}\left(\Omega, \mathbb{R}^{2}, \beta\right) \times \mathbb{P}_{\mathbb{R}}\right]_{\infty}$ such that

$$
\Psi\left(a_{0}, a_{1}, a_{2}, a_{3}\right)=\#_{\hat{\gamma}} \psi\left(a_{0}, a_{1}, a_{2}, a_{3}\right)
$$

for $a_{0}, \ldots, a_{3}$ in the subalgebra $\left(C^{\infty}\left(\Omega, \mathbb{R}^{2}, \beta\right) \times, \mathbb{R}\right)^{\infty}$. It is obvious that we have an inclusion map

$$
l:\left(\left(\left[C(\Omega) \times{ }_{\theta} \mathbb{R}\right]_{\infty} \times{ }_{\gamma} \mathbb{R}\right)^{\infty} \times{ }_{\hat{\gamma}} \mathbb{R}\right)^{\infty} \rightarrow\left[C^{*}\left(\Omega, \mathbb{R}^{2}, \beta\right) \times{ }_{\hat{\gamma}} \mathbb{R}\right]_{\infty},
$$

which induces a homomorphism $l_{*}$ between the corresponding $K$-groups.

Lemma 4.4. The map

$$
l_{*} T_{\gamma_{*}} r_{*}: K_{1}\left(\left[C(\Omega) \times{ }_{\theta} \mathbb{R}\right]_{\infty}\right) \rightarrow K_{1}\left(\left[C^{*}\left(\Omega, \mathbb{R}^{2}, \beta\right) \times{ }_{\hat{\gamma}} \mathbb{R}\right]_{\infty}\right)
$$

is a surjective homomorphism.

Proof. Let $h_{j}$ be $j^{\text {th }}$ Hermite function, $j=0,1,2, \ldots$, and let $e_{j k}$ be the rank-one operator $h_{j} \otimes h_{k}\left(\in \mathscr{K}^{\infty}\right)$ on $L^{2}(\mathbb{R})$ [17]. Let $A_{n}$ be the algebra generated by $e_{j k}, j, k=0,1, \ldots, n$. For any $n$, the map $a \mapsto a \otimes e_{00}$ induces a surjective homomorphism from $K_{1}\left(\left[C(\Omega) \times{ }_{\theta} \mathbb{R}\right]_{\infty}\right)$ onto $K_{1}\left(\left[C(\Omega) \times{ }_{\theta} \mathbb{R}\right]_{\infty} \otimes A_{n}\right)$. It is easy to see that $\bigcup_{n=0}^{\infty} T_{\gamma}\left(\left[C(\Omega) \times{ }_{\theta} \mathbb{R}\right]_{\infty} \otimes A_{n}\right)$ is dense (in the $C^{*}$-norm) in $\left(\left(\left[C(\Omega) \times{ }_{\theta} \mathbb{R}\right]_{\infty} \times\right.\right.$ $\left.\left.{ }_{\gamma} \mathbb{R}\right)^{\infty} \times{ }_{\hat{\gamma}} \mathbb{R}\right)^{\infty}$. Because $\left[C^{*}\left(\Omega, \mathbb{R}^{2}, \beta\right) \times{ }_{\hat{\gamma}} \mathbb{R}\right]_{\infty}$ is closed under holomorphic functional calculus, to complete the proof, it suffices to show that if $B \in\left(\left[C(\Omega) \times{ }_{\theta} \mathbb{R}\right]_{\infty} \otimes\right.$ $\left.A_{n}\right)^{\sim} \otimes M_{k}$ is invertible in $\left(\left[C(\Omega) \times{ }_{\theta} \mathbb{R}\right] \otimes A_{n}\right)^{\sim} \otimes M_{k}$, then $B^{-1} \in\left(\left[C(\Omega) \times{ }_{\theta} \mathbb{R}\right]_{\infty} \otimes\right.$ $\left.A_{n}\right)^{\sim} \otimes M_{k}$. But this is obvious because, by the definition of $\left[C(\Omega) \times{ }_{\theta} \mathbb{R}\right]_{\infty}$, for any $b \in\left[C(\Omega) \times{ }_{\theta} \mathbb{R}\right]_{\infty} \otimes A_{n} \otimes M_{k}$ with $C^{*}$-norm less than $1, \sum_{j=1}^{\infty} b^{j} \in\left[C(\Omega) \times{ }_{\theta} \mathbb{R}\right]_{\infty} \otimes$ $A_{n} \otimes M_{k}$.

Proof of Theorem 3.3. By [17, Theorem 6.2], we have, for any $[P] \in K_{0}\left(C^{\infty}\left(\Omega, \mathbb{R}^{2}, \beta\right)\right)$, $\langle[\psi],[P]\rangle=\left\langle\left[\#_{\hat{\gamma}} \psi\right], \phi_{\hat{\gamma}, 0}[P]\right\rangle$, which, according to [15, p. 109] and (4.6), equals $\left\langle[\Psi], \phi_{\hat{\gamma}, 0}[P]\right\rangle$. According to Lemma $4.4, \phi_{\hat{\gamma}, 0}[P]$ can be represented by $T_{\gamma *} r_{*} v$ with $v \in K_{1}\left(\left[C(\Omega) \times{ }_{\theta} \mathbb{R}\right]_{\infty}\right)$. Because of (4.6) and (4.7), we have $\langle[\psi],[P]\rangle=\langle[\Psi]$, $\left.T_{\gamma *} r_{*} v\right\rangle=\left\langle[\# \psi], T_{\gamma *} r_{*} v\right\rangle$. Since, by (4.4), the restriction of $\psi$ to $([C(\Omega) \times$ $\left.\left.{ }_{\theta} \mathbb{R}\right]_{\infty} \times{ }_{\gamma} \mathbb{R}\right)^{\infty}$ is $-\#_{\gamma} \Lambda_{\theta}$, the restriction of $\#_{\hat{\gamma}} \psi$ to $\left(\left(\left[C(\Omega) \times{ }_{\theta} \mathbb{R}\right]_{\infty} \times{ }_{\gamma}[\mathbb{R})^{\infty} \times{ }_{\hat{\gamma}} \mathbb{R}\right)^{\infty}\right.$ equals $-\#_{\hat{\gamma}} \#_{\gamma} \Lambda_{\theta}$. Therefore $\langle[\psi],[P]\rangle=-\left\langle \#_{\hat{\gamma}} \#_{\gamma}\left[\Lambda_{\theta}\right], T_{\gamma *} r_{*} v\right\rangle[15$, p. 109]. By Lemmas 4.1 and 4.3 , we have $\langle[\psi],[P]\rangle=-\left\langle\left[\Lambda_{\theta}\right], v\right\rangle=-\left\langle[\mu], \phi_{\theta, 0}^{-1} v\right\rangle$. To 
prove $v=\phi_{\gamma, 1}^{-1}[P]$, it suffices to consider the $K$-groups of the $C^{*}$-algebras involved here. Since $\phi_{\hat{\gamma}, 0} \phi_{\gamma, 1}=T_{\gamma *} r_{*}$ on $K_{1}\left(C(\Omega) \times{ }_{\theta} \mathbb{R}\right)[13]$, the equation $T_{\gamma *} r_{*} v=\phi_{\hat{\gamma}, 0}[P]$. implies $\phi_{\gamma, 1} v=[P]$. Hence, by (3.7),

$$
\langle[\psi],[P]\rangle=-\left\langle[\psi], \phi_{\theta, 0}^{-1} \phi_{\gamma, 1}^{-1}[P]\right\rangle=-\left\langle[\mu], \phi^{-1}[P]\right\rangle .
$$

Acknowledgements. The author wishes to thank Professor T. Natsume for his communication of [17], which plays an essential role in the proof of our main result, and for discussions related to this work. The author is greatly indebted to Professor J. Pincus. It was he who led the author to the study of differential operators associated to disordered systems; without his guidance this work would not be possible. The author also wishes to thank Professor J. Bellissard for communicating his work on the quantum Hall effect and for his comments on an early draft of the paper.

\section{References}

1. Avron, J., Seiler, R.: Quantization of the Hall conductance for general multiparticle Schrödinger Hamiltonians. Phys. Rev. Lett. 54, 259-262 (1985)

2. Avron, J., Seiler, R., Simon, B.: Homotopy and quantization in condensed matter physics. Phys. Rev. Lett. 51, $51-53$ (1983)

3. Avron, J., Seiler, R., Yaffe, L.: Adiabatic theorems and applications to the quantum Hall effect. Commun. Math. Phys. 110, 33-49 (1987)

4. Bellissard, J.: Schrödinger operators with an almost periodic potential. Lecture Notes in Physics, Vol. 153, Berlin, Heidelberg, New York: Springer 1982

5. Bellissard, J.: $K$-Theory of $C^{*}$-algebras in solid state physics. Statistical mechanics and field theory, Mathematical aspects. Dorlas, T., Hugenholtz, M., Winnink, M. (eds.). Lecture Notes in Physics, Vol. 257, pp. 99-156 Berlin, Heidelberg, New York: Springer 1986

6. Bellissard, J.: Ordinary quantum Hall effect and noncommutative cohomology. Lecture Notes, 1986

7. Bellissard, J.: $C^{*}$-Algebra in solid state physics, $2 D$ electrons in a uniform magnetic field. Lecture Notes, 1987

8. Bellisard, J.: Private communication, 1988

9. Bellisard, J., Lima R., Testard, D.: Almost periodic Schrödinger operators, in "Mathematics + Physics”, 1, pp. 1-64. Streit, L. (ed), Philadelphia, Singapore: World Scientific 1985

10. Blackadar, B.: K-Theory for Operator Algebras, MSRI Publications, 1985

11. Claro, F., Wannier, W.: Magnetic subband structure of electrons in hexagonal lattices. Phys. Rev. B19, 6068-6074 (1979)

12. Coburn, L., Moyer, R., Singer, I.: $C^{*}$-Algebras of almost periodic pseudo-differential operators. Acta Math. 139, 279-307 (1973)

13. Connes, A.: An analogue of the Thom isomorphism of $C^{*}$-algebras by an action of $\mathbb{R}$. Adv. Math. 39, 31-55 (1981)

14. Connes, A.: A survey of foliations and operator algebras. Proceedings of Symposia in Pure Mathematics, Vol. 38, pp. 521-628. Providence, RI: AMS 1982

15. Connes, A.: Noncommutative differential geometry. Publ. Math. I.H.E.S., 62, 257-360 (1986)

16. Elliott, G.: Gaps in the spectrum of an almost periodic Schrödinger operator. C.R. Math. Rep. Acad. Sci. Canada 4, 255-259 (1982)

17. Elliott, G., Natsume, T., Nest, R.: Cyclic cohomology for one parameter smooth crossed products. Acta Math. (to appear)

18. Gudmundsson, V., Gerhardts, R.: Interpretation of experiments implying density of states between Landau levels of a two-dimensional electron gas by a statistical model for inhomogeneity, Phys. Rev. B35, 8005-8014 (1987)

19. Halperin, B.: Quantized Hall conductance current-carrying edge states, and the existence of extended states in a two-dimensional disordered potential. Phys. Rev. B25, 2185 (1982)

20. Kaminker, J., Xia, J.: The spectrum of operators elliptic along the orbits of $\mathbb{R}^{n}$ action. Commun. Math. Phys. 110, 427-438 (1987) 
21. von Klitzing, K., Dorda, G., Pepper, M.: Realization of a resistance standard based on fundamental constants. Phys. Rev. Lett. 45, 494-497 (1980)

22. Kunz, H.: The quantum Hall effect for electrons in a random potential. Commun. Math. Phys. 112, 121-145 (1987)

23. Laughlin, R.: Quantized Hall conductivity in two dimensions. Phys. Rev. B23, 5632-5634 (1981)

24. Mott, N., Jones, H.: The Theory of the Properties of Metals and Alloys. Oxford, University Press 1962

25. Naimark, M.: Linear differential operators, part II, linear differential operators in Hilbert space. New York: Frederick Ungar Publishing 1968

26. Pedersen, G.: $C^{*}$-Algebras and their automorphism groups. London: Academic Press 1979

27. Shubin, M.: The spectral theory and the index of elliptic operators with almost periodic potential. Russ. Math. Surv. 34, 109-157 (1979)

28. Thouless, D. Kohmoto, M., Nightingale P., Deh-Nijs, M.: Quantized Hall conductance in a two-dimensional periodic potential. Phys. Rev. Lett. 49, 405-408 (1982)

Communicated by B. Simon

Received November 11, 1987; in revised form March 8, 1988 